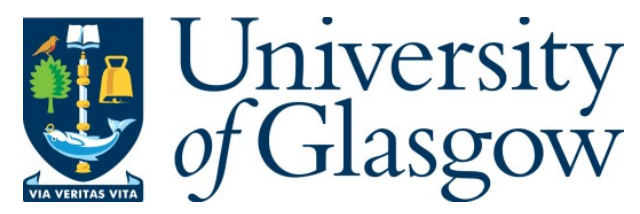

Carrion, M., Steijl, R., Barakos, G., and Stewart, D. (2016) Analysis of hybrid air vehicles using computational fluid dynamics. Journal of Aircraft, 53(4), pp. 10011012.

There may be differences between this version and the published version. You are advised to consult the publisher's version if you wish to cite from it.

http://eprints.gla.ac.uk/116454/

Deposited on: 18 March 2016

Enlighten - Research publications by members of the University of Glasgow http://eprints.gla.ac.uk 


\title{
Analysis of Hybrid Air Vehicles using Computational Fluid Dynamics
}

\author{
M. Carrión; R. Steijl† G.N. Barakos $\ddagger$ D. Stewart ${ }^{\S}$
}

\begin{abstract}
This paper presents a study of the aerodynamics of shapes pertinent to lighter-than-air vehicles, using computational fluid dynamics. The work begins with the validation of the computational-fluid-dynamics method using a 6:1 prolate spheroid. The validated method is then employed for the study of the flow around a shape similar to the Airlander 50 airship of Hybrid Air Vehicles Ltd. An overview of the flow around this kind of shape is presented, supported by pressure survey, flow visualisation and transition effects, as function of the Reynolds number. The sensitivity of the transition location to the Reynolds number is also demonstrated, and then, the role of each component of the vehicle is analysed. The effect of each component on the flow-field, the lift and drag, and stability in pitch are provided. It was found that the fins contributed the most to increase the lift and drag coefficients.
\end{abstract}

\section{Nomenclature}

$\begin{array}{llll}C_{D} & =\text { drag coefficient } & \mathbf{R} & =\text { residual } \\ C_{f} & =\text { friction coefficient } & R e & =\text { Reynolds number } \\ C_{L} & =\text { lift coefficient } & \mathbf{S} & =\text { source vector in Navier-Stokes equations } \\ C_{M} & =\text { pitching moment } & U & =\text { velocity vector } \\ C_{p} & =\text { pressure coefficient } & u_{\infty} & =\text { freestream velocity, } \mathrm{m} / \mathrm{s} \\ D & =\text { drag } & V & =\text { cell volume } \\ d & =\text { vehicle diameter, } \mathrm{m} & x & =\text { axial position, } \mathrm{m} \\ h & =\text { helicity, } \mathrm{m}^{2} / \mathrm{s}^{2} & x, y, z & =\text { Cartesian coordinates } \\ L & =\text { vehicle length, } \mathrm{m} & \alpha & =\text { pitch angle, deg } \\ M_{\infty} & =\text { freestream Mach number } & \Omega & =\end{array}$

\section{Introduction}

The study of lighter than air (LTV) and hybrid air vehicles ${ }^{11}$ (HAV) involves a number of challenges. Firstly, these type of vehicles are characterised by flying at low speeds, while having very high Reynolds number. In both the wind tunnel and CFD aerodynamic studies of these type of aircraft the Reynolds number is a key issue, as it can change up to two orders of magnitude from the tunnel models to full scale

\footnotetext{
*Research Associate, CFD Laboratory, School of Engineering; marina.carrion@glasgow.ac.uk

${ }^{\dagger}$ Lecturer, CFD Laboratory, School of Engineering; rene.steijl@glasgow.ac.uk

${ }^{\ddagger}$ Professor, MAIAA, MRAeS, CFD Laboratory; george.barakos@glasgow.ac.uk

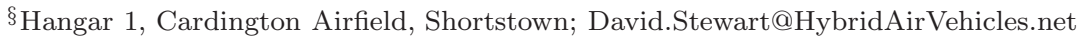


aircraft. Since favorable pressure gradients are present on the vehicle's surface, the boundary layer tends to be laminar. It therefore stays laminar or becomes turbulent with the decrease/increase of Reynolds number. In addition, due to their shape, there is flow separation at the back of the vehicle.

Over the past years, a few projects have been undertaken on the development of LTV technology. One example is the ZHIYUAN-1 stratospheric airship ${ }^{2}$ developed in China. In the UK, the YEZ-2A project ${ }^{3}$ was launched to demonstrate the capability of airships as airborne early warning systems for the US Navy. Currently, the LOCATE project (Collaborative industrial research into technology and manufacturing capability of Low Carbon aircraft using lighter than air Technology) is aiming to innovative novel configuration of LTV vehicles.

Computational Fluid Dynamics (CFD) is also emerging as a tool for the design and optimisation of LTV. Suman et al ${ }^{[4}$ studied the bare hull of the ZHIYUAN-1 stratospheric airship ${ }^{2}$ with the unstructured TAU solver of DLR. They used Reynolds Averaged Navier-Stokes (RANS) in their computations and fixed the transition point by suppressing the turbulence model in the laminar region. Sensitivity studies on the transition location showed a decrease in drag when it was moved downstream up to $75 \% \mathrm{~L}$, due to the presence of laminar flow. From that point, the drag increased with distance, due to the presence of separated flow. The numerical results with fixed transition at $52 \% \mathrm{~L}$ agreed broadly with the experimental data for angles of attack up to 5 deg. However, poor agreement was found for free-transition cases and for those with fixed transition at angles of attack higher than $5 \mathrm{deg}$. These discrepancies were attributted to the RANS method

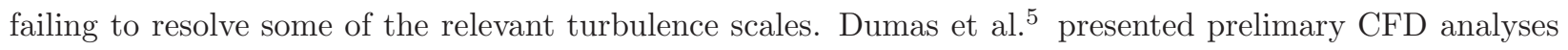
of an open volume airship concept within the MAAT (Multibody Advanced Airship for Transport) project.

For validation and development of numerical methods related to LTVs, prolate spheroids have been extensively employed. There is experimental data availabe in the open literature for a $6: 1$ prolate spheroid 6 for a wide range of flow conditions and for a 3:1 prolate spheroid ${ }^{7}$ Sorenser ${ }^{8}$ employed the $6: 1$ prolate spheroid ${ }^{\sqrt{6}}$ for the validation of the $\gamma-R_{\theta}$ transition model, for a range of Reynolds numbers between 3.2 and 9.6 million and at zero and 30 degrees of incidence. For the pressure, fully turbulent and transition cases showed good agreement with the experiments. Although at zero incidence the transition model agreed better with the experimental friction coefficients than the fully turbulent case, the $\gamma-R e_{\theta}$ model was not able to predict the correct location of transition at $30 \mathrm{deg}$. This was attributed to the lack of cross-flow transition prediction capability of the employed transition model. Similar studies were performed by other authors $\frac{9}{10}$ In the case of airships, the 6:1 prolate spheroid was also employed to approximate the shape of the hull by Omari et al 11,12

To the best of the authors' knowledge, a CFD study of the complete configuration of an LTV or HAV has not been presented in the literature. Hence, after validating the employed CFD methods of Hybrid Air Vehicles Ltd., the present paper provides a study of the aerodynamics for an early variant of the Airlander $50 \mathrm{HAV}$.

\section{Numerical Method}

The Helicopter Multi-Block (HMB2) CFD solver, $\frac{13}{13}$ developed at Liverpool, is used for the present work, and has so far been validated for a number of applications, including helicopters, wind turbines, turboprop and unmannned combat aerial vehicle aircraft. HMB2 solves the Navier-Stokes equations in integral form 
using the Arbitrary Lagrangian Eulerian formulation for time-dependent domains with moving boundaries:

$$
\frac{d}{d t} \int_{V(t)} \mathbf{w} d V+\int_{\partial V(t)}\left(\mathbf{F}_{\mathbf{i}}(\mathbf{w})-\mathbf{F}_{v}(\mathbf{w})\right) \mathbf{n} d S=\mathbf{S}
$$

where $V(t)$ is the time dependent control volume, $\partial V(t)$ its boundary, w is the vector of conserved variables $[\rho, \rho u, \rho v, \rho w, \rho E]^{T} . \mathbf{F}_{i}$ and $\mathbf{F}_{v}$ are the inviscid and viscous fluxes, including the effects of the mesh movement.

The Navier-Stokes equation are discretised using a cell-centred finite volume approach on a multi-block grid, leading to the following equations:

$$
\frac{\partial}{\partial t}\left(\mathbf{w}_{i, j, k} V_{i, j, k}\right)=-\mathbf{R}_{i, j, k}\left(\mathbf{w}_{i, j, k}\right)
$$

where $\mathbf{w}$ represents the cell variables and $\mathbf{R}$ the residuals. $i, j$ and $k$ are the cell indices and $V_{i, j, k}$ is the cell volume. Osher's 14 upwind scheme is typically used for the discretisation of the convective terms and MUSCL ${ }^{15}$ variable extrapolation is used to provide a formally $3 \mathrm{rd}$ order accurate scheme. To account for low-speed flows, the Low-Mach Roe scheme (LM-Roe) developed by Rieper ${ }^{16}$ is employed $!^{17}$ The linearised system is solved using the generalised conjugate gradient method with a block incomplete lower-upper (BILU) pre-conditioner!18 The HMB2 solver has a library of turbulence closures which includes several oneand two- equation turbulence models and versions of the $k-\omega$ model, including the recently developed Scale-Adaptative Simulation (SAS) model, $\frac{19}{19}$ and the $\gamma-R e_{\theta_{t}}$ transition model $[20$

\section{Mesh Generation}

Multi-block structured topologies were employed, to allow for a good representation of the arship surface. The blocks are also used to allow for easy sharing of the computational load between processors for parallel computing. Since the vehicle is symmetric with respect to the XZ plane, only half of the vehicle was meshed, assuming symmetry. This reduced the size of the grid by half and therefore enabled savings on computational resources. Nevertheless, some cases were computed on the full mesh to ascertain that the symmetry assumption appears to be valid if the flow yaw angle is zero. At the inflow, outflow and far-field boundaries free-stream conditions were assumed, as can be seen in Figure 1(a).

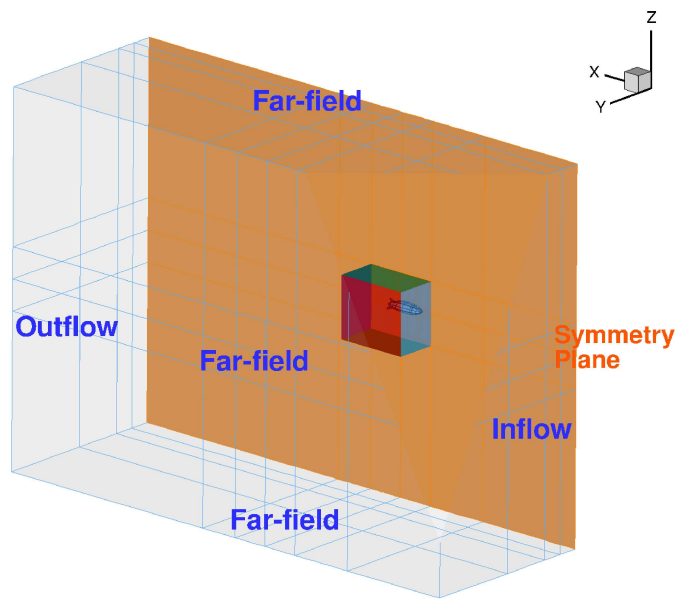

(a) Domain extension and boundaries.

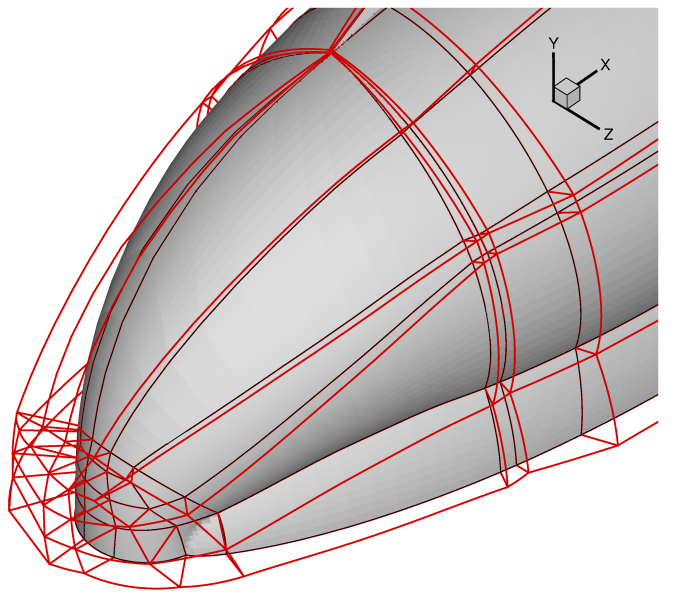

(b) O-topology around the body.

Figure 1: Overview of the multi-block topology for the Airlander 50. 


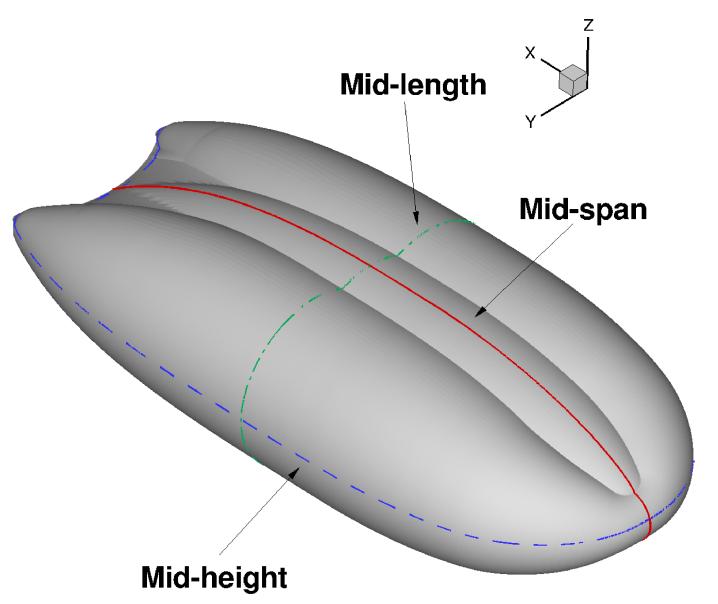

(a) AL50 1: Bare hull.

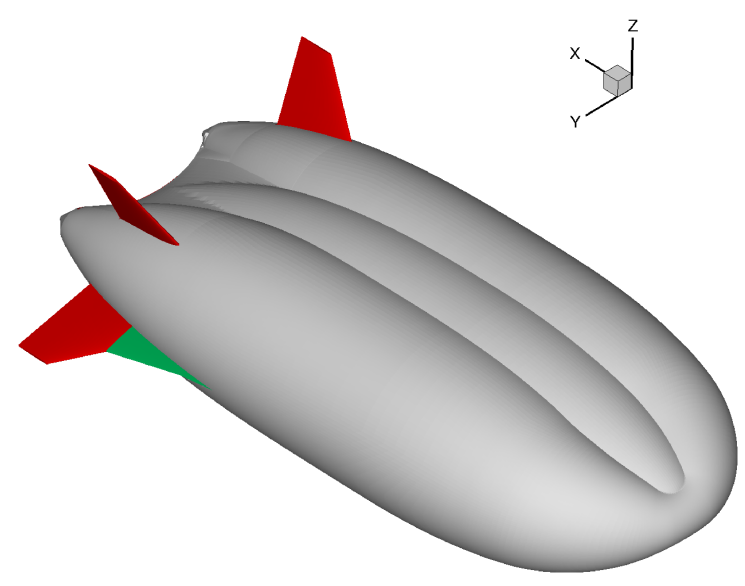

(c) AL50 3: Hull, fins \& LERX.

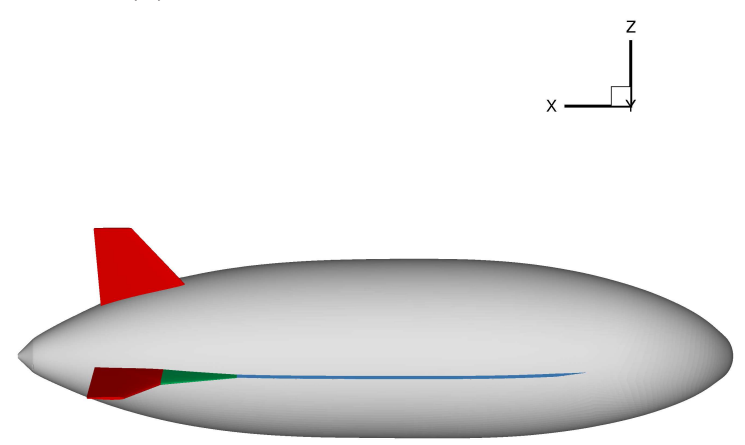

(e) Side view of configuration AL50 4 .

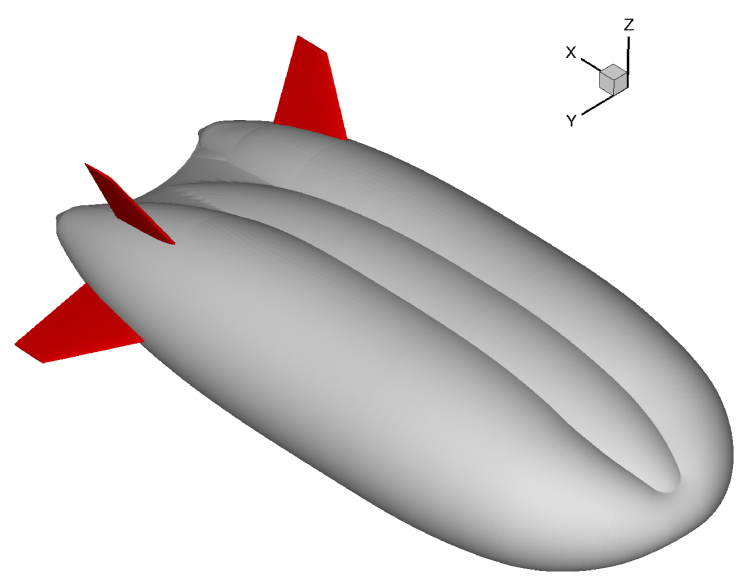

(b) AL50 2: Hull \& fins.

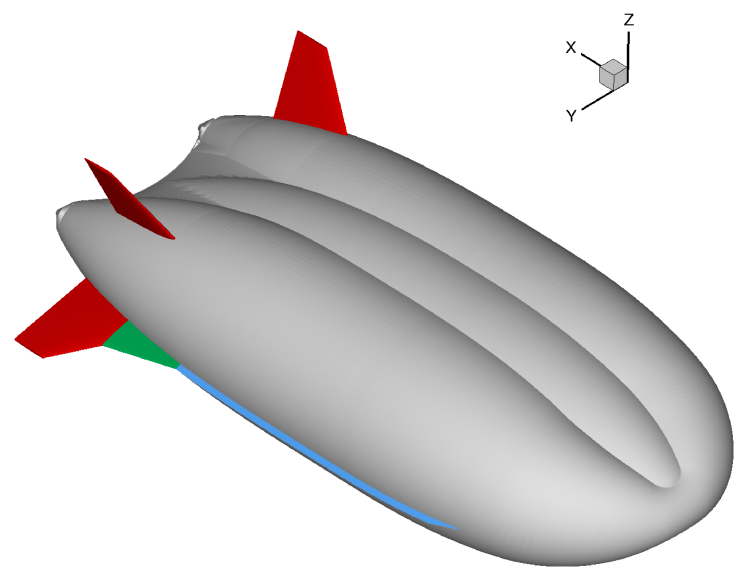

(d) AL50 4: Hull, fins, LERX \& strakes

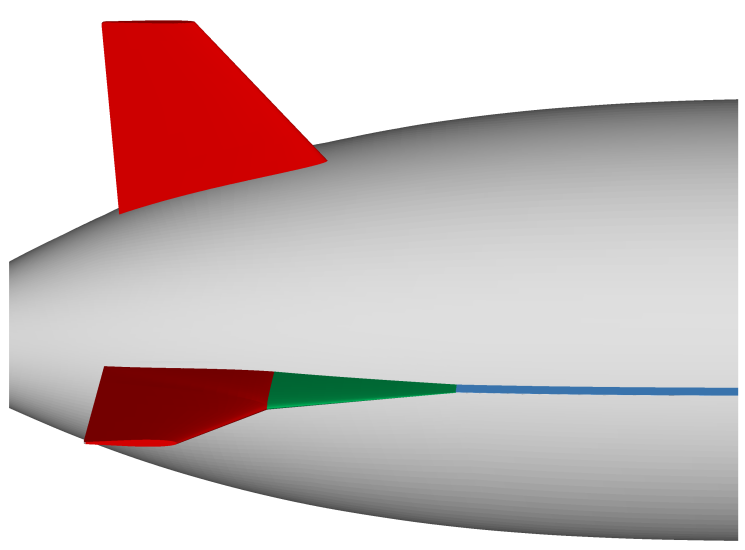

(f) Zoom of side view of configuration AL50 4.

Figure 2: Vehicle configurations. 
A set of five sliding planes 21 were employed, to allow for localised grid refinement near the body and to have a cartesian grid in the rest of the domain. These planes are the ones close to the body presented in Figure 1 (a). An O-topology was employed around the Airlander 50, for optimal orthogonality of the cells to the surface, see Figure 1 (b). A first cell size of $3 \mu \mathrm{m}$ was employed, to ensure a $y^{+}$of $<1$.

Four configurations were considered for the Airlander 50 vehicle, which are shown in Figure 2 and whose properties are summarised in Table 1. AL50 1 consists of the bare hull; the fins were then added (AL50 2) and also the leading edge root extension (LERX) in configuration AL50 3. Finally, the strakes were considered in configuration AL50 4. For the full configuration, side views are presented in Figures 2 (e) and (f). To assess grid convergence, two grid refinements were employed for the baseline case (grids AL50 4 and AL50 5).

Table 1: Summary of mesh properties.

\begin{tabular}{cccccc}
\hline Grid & Components & $\begin{array}{c}\text { Size } \\
(\text { Blocks })\end{array}$ & $\begin{array}{c}\text { Cross sect.- } \\
(\text { span)-wise cells }\end{array}$ & $\begin{array}{c}\text { Boundary } \\
\text { layer cells }\end{array}$ & $\begin{array}{c}\text { Boundaries } \\
\text { in L }\end{array}$ \\
\hline AL50 $\mathbf{1}$ & Bare Hull & $9.2 \mathrm{M}(1,069)$ & $236(314)$ & 34 & I:5 O:8 FF:5 \\
AL50 2 & Hull \& Fins & $9.5 \mathrm{M}(1,151)$ & $236(314)$ & 34 & I:5 O:8 FF:5 \\
AL50 $\mathbf{3}$ & Hull, Fins \& LERX & $10.8 \mathrm{M}(1,203)$ & $236(314)$ & 34 & I:5 O:8 FF:5 \\
AL50 4 & Hull, Fins, LERX \& Strakes & $10.8 \mathrm{M}(1,203)$ & $236(314)$ & 34 & I:5 O:8 FF:5 \\
AL50 5 & Refined AL50 4 & $28.4 \mathrm{M}(1,253)$ & $456(410)$ & 60 & I:5 O:8 FF:5 \\
\hline
\end{tabular}

AL50: Airlander 50, L: Length, I: Inflow, O: Outflow, FF: Far-field. Size of mesh in million cells.

\section{Results and discussion}

The computational results are presented in this section. Since no experimental data is available for the Airlander 50, as an early stage design was employed, and the hull has some resemblance to a spheroid, the 6:1 prolate spheroid ${ }^{22}$ was employed for the validation of the method. A 20-degree pitched case was studied, at Reynolds number of 4.2 million and Mach number of 0.15. A number of computations are then presented for the approximate Airlander 50 (AL50), including the role of the strakes in the aerodynamic coefficients and effect of the flow conditions and Reynolds number on the transition onset. In all cases, a wind speed of $40 \mathrm{~m} / \mathrm{s}$ was employed and sea level flow conditions were assumed. Unless otherwise specified, a Reynolds number, based on the vehicle's length (L) of 3 million was employed. RANS and URANS computations were performed, with the k- $\omega$ SST turbulence model by Menter.23

\section{A. Validation of the method}

For the 6:1 prolate spheroid, 22 an O-topology was employed for the grid of 40 million cells, as shown in Figure 3 .

Two main counter-rotating vortices are generated, as can be seen in the contours of the $Q-$ criterion 24 of Figure 4 (a). A secondary vortex is also captured, located at an azimuth of 140 degrees, which is in good agreement with experiments $\sqrt[22]{ }$ The rotation of the secondary vortex is counter to the rotation of the main vortex. 

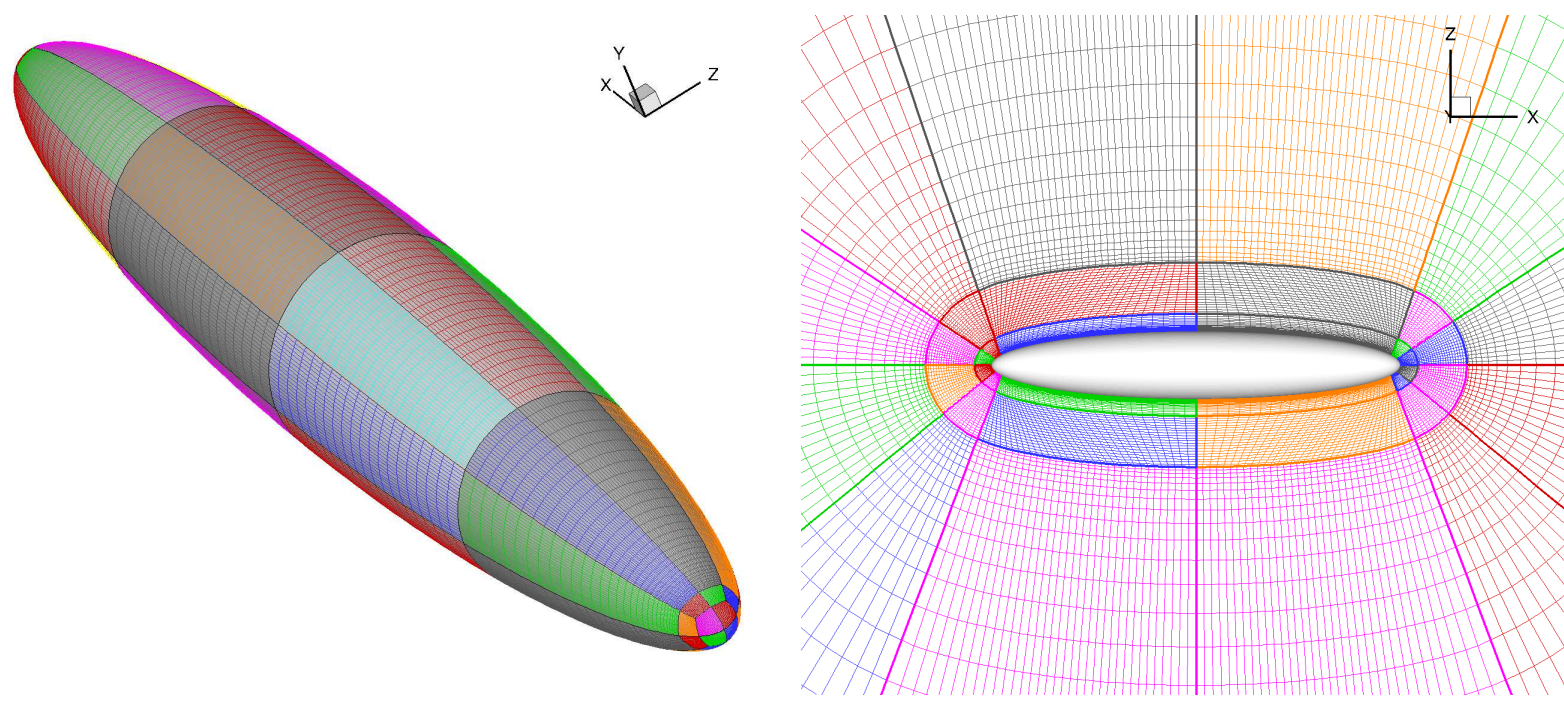

Figure 3: Multi-block mesh for the 6:1 prolate spheroid.

The correct prediction of the flow can also be seen in the surface pressure coefficient distribution of Figure 四(b). At $x=77 \% \mathrm{~L}$, the presence of the second vortex very close to the spheroid surface leads to a drop in pressure, with very good agreement with the experimental data published by Wetzel et al $l^{6}$ In the case of the friction coefficient $\left(C_{f}\right)$ presented in Figure $4(\mathrm{c})$, the experiments by Chesnakas et al ${ }^{[22}$ were employed. In this case, reasonable agreement with experiments was obtained from 90 to 150 deg. of azimuth. From 150 to 180 deg., however, the CFD under-predicted the $C_{f}$ peak values. Similar agreement was obtained to other works presented in the literature 10 who also predicted less peak-to-peak variation, even though they employed different turbulence models (RANS and DES with Spalart-Allmaras). These discrepancies in friction could be due to difficulties of the turbulence models to correctly predict the shedding of the vortical structure, which seem to be weaker in the CFD, but also to difficulties in the experiments to measure these quantitates with high level of accuracy. In addition, it should be noted that in the experiments $\frac{22}{2}$ the friction coefficient was not measured directly, but a curve fit was employed instead. Nevertheless, the CFD predicted successfully the trend of the experimental data.

Figure 5 shows contours of helicity on a slice at $x=77 \% \mathrm{~L}$. Helicity is obtained by the dot product of the vorticity and the velocity vectors, $h=U \cdot(\nabla \times U)$. For these flow quantities, good agreement with the experiments 22 is also observed.

\section{B. Flow topology around an airship}

The flow around the 'AL50' is studied here, including the surface pressure, vortex generation and transition effects.

\section{Pressure field analysis}

A comparison is first shown between the AL50 4 and AL50 5 cases of Table 1 . The pressure coefficient $\left(C_{p}\right)$ at the symmetry and mid-height planes are presented in Figure 6 Some variations in $C_{p}$ are observed at the nose of the vehicle (Figure 6 (a)), which are due to the sudden change of curvature in that region, as 


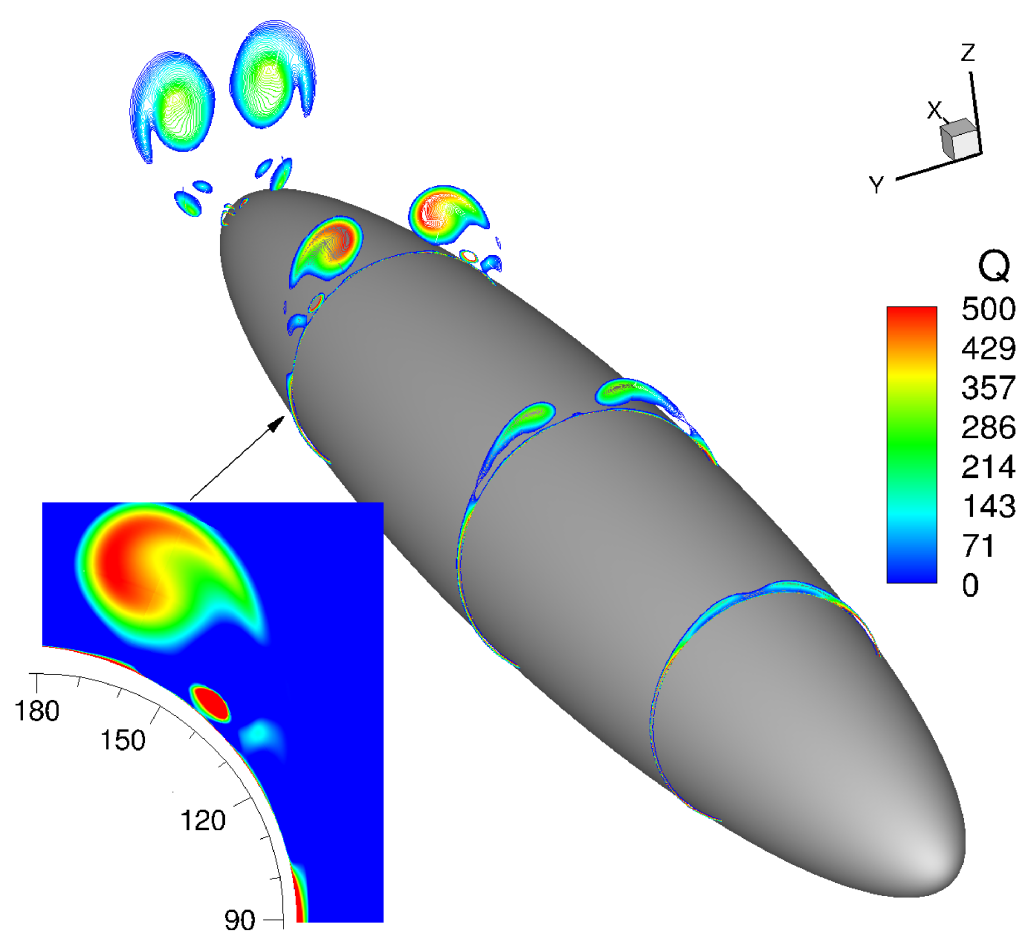

(a) Contours of $Q$-criterion, 24 with detail at $x=77 \% \mathrm{~L}$.

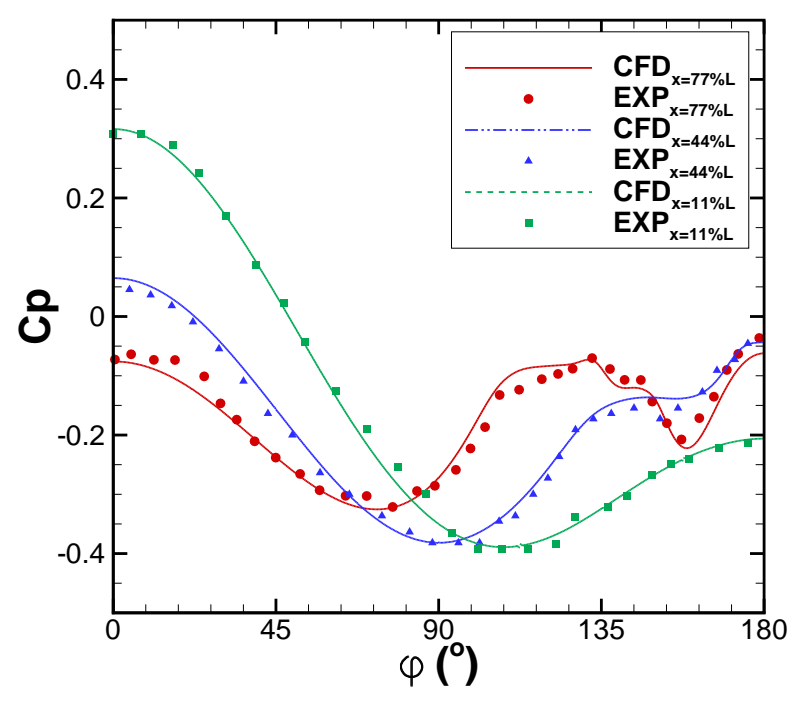

(b) Pressure coefficient at three axial stations.

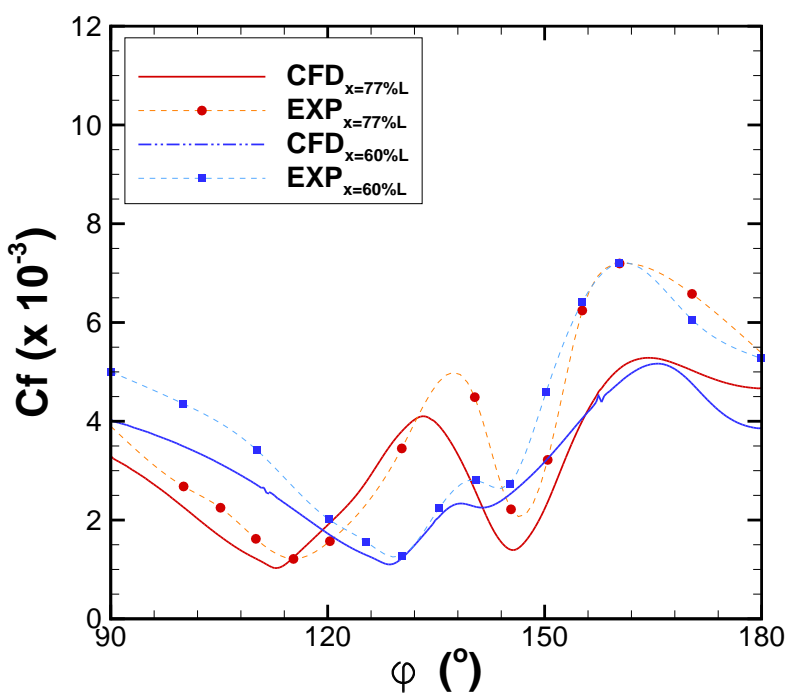

(c) Friction coefficient at two axial stations.

Figure 4: Flow around a 6:1 prolate spheroid at $\alpha=20^{\circ}, M_{\infty}=0.15$ and $R e=4.2 \cdot 10^{6}$.

shown in Figure 2 (a). In the slice studied in Figure 6 (b) this is not observed. The solutions obtained with both grids are practically the same, with small differences close to the back of the vehicle. Grid convergence can therefore be assumed, with the employed URANS framework.

As can be observed in Figure [6 favourable pressure gradients $(\partial p / \partial s<0)$ are present from the nose to $25 \% \mathrm{~L}$, which indicates fully attached flow. From 25 to $80 \% \mathrm{~L}$, a neutral pressure gradient $(\partial p / \partial s \approx 0)$ is 


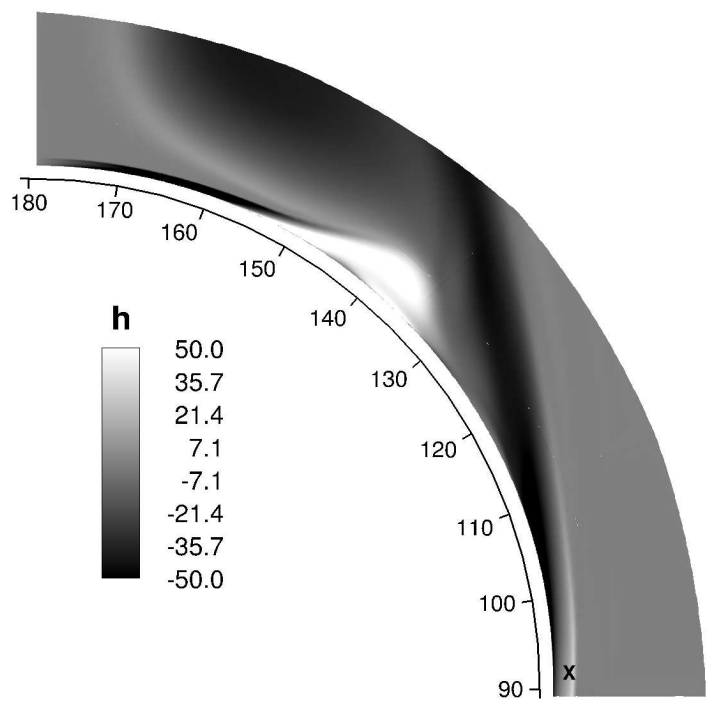

(a) CFD.

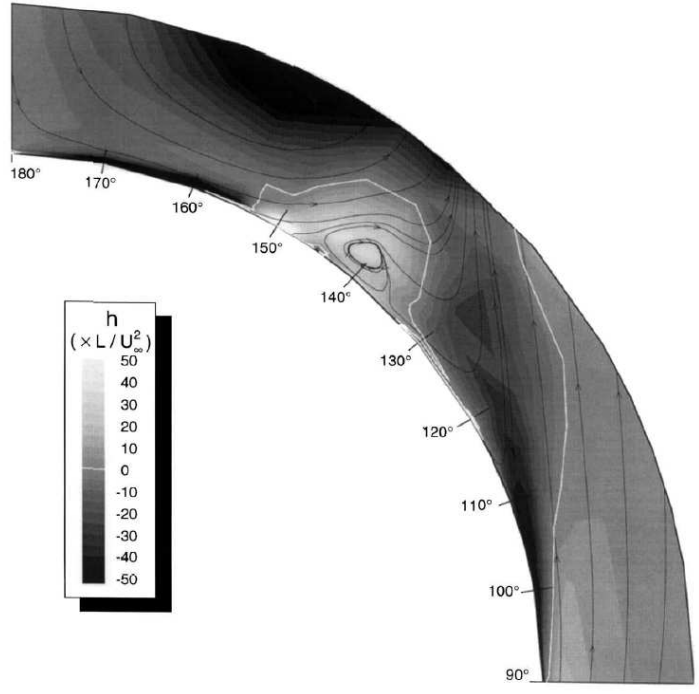

(b) Experiments. $[22$

Figure 5: Flow around a 6:1 prolate spheroid at $\alpha=20^{\circ}, M_{\infty}=0.15$ and $R e=4.2 \cdot 10^{6}$. Contours of helicity at at $x=77 \% L$.

present in both upper and lower surfaces. From $80 \% \mathrm{~L}$, the adverse pressure gradient $(\partial p / \partial s>0)$ leads to flow separation. The effect of each of the vehicle's components to the overall loads is further analysed in Section D

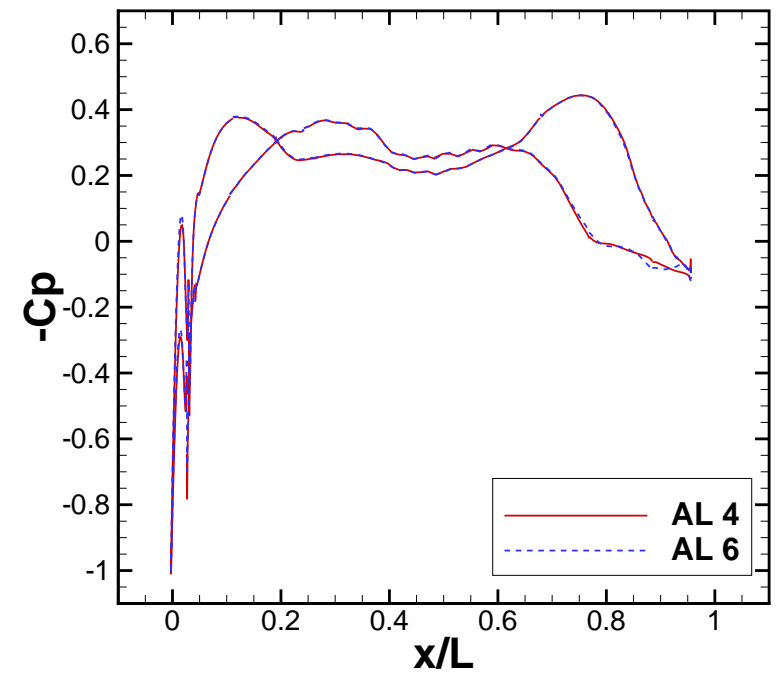

(a) Symmetry plane.

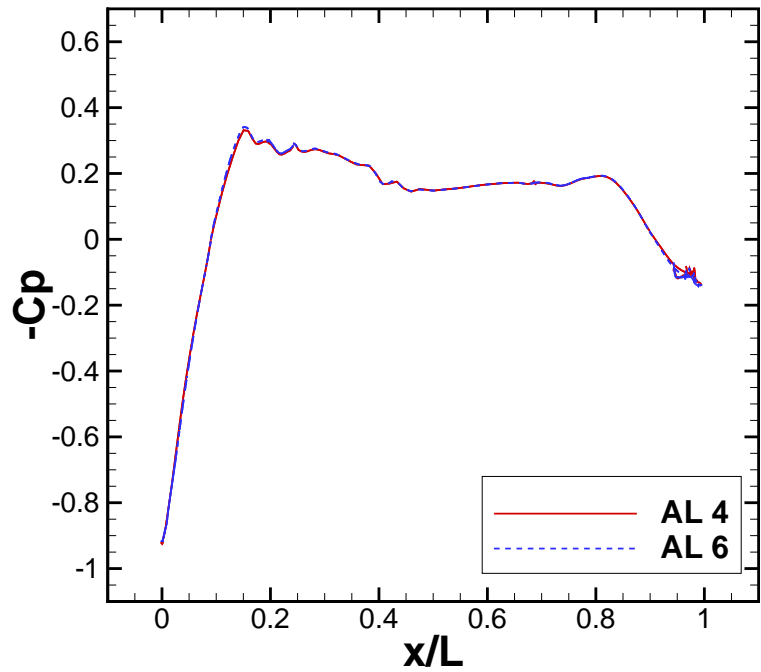

(b) Mid-height plane.

Figure 6: Flow around the approximated Airlander 50 at $\alpha=0^{\circ}, M_{\infty}=0.12$ and $R e=3 \cdot 10^{6}$. Effect of the mesh density in the $C_{p}$. 


\section{Flow visualisation}

Figure 7 shows the vortices generated by the different components of the airship, for 10 and 20 degrees of pitch. As can be observed, a clock-wise vortex is firstly formed by the strake (A), see Figures 7 (a) and (d), with higher strength at the higher pitch angle. Additionally, the curvature of the hull leads to the creation of an upper vortex $(\mathbf{B})$, that appears at $x=0.60 \mathrm{~L}$, approximately. This is more obvious for the 20-degree pitched case.

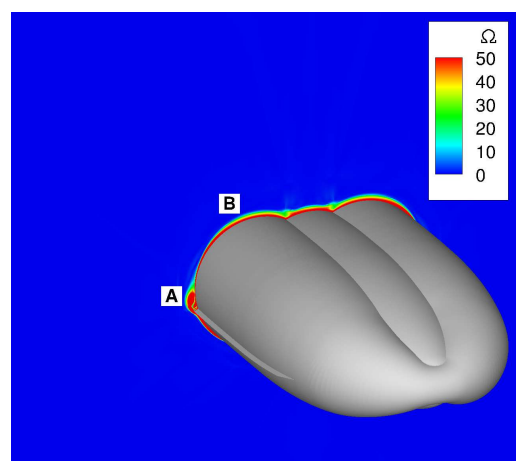

(a) $x=0.60 \mathrm{~L}, \alpha=10 \mathrm{deg}$.

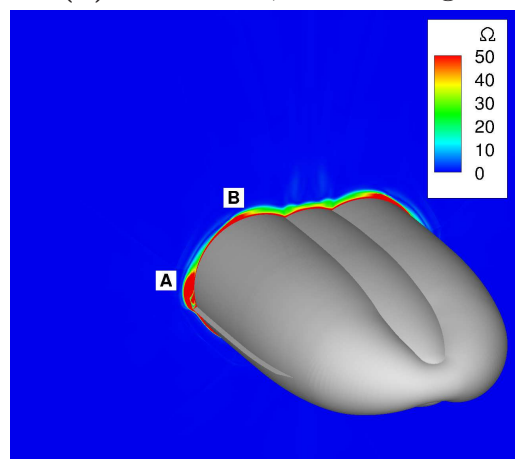

(d) $x=0.60 \mathrm{~L}, \alpha=20 \mathrm{deg}$.

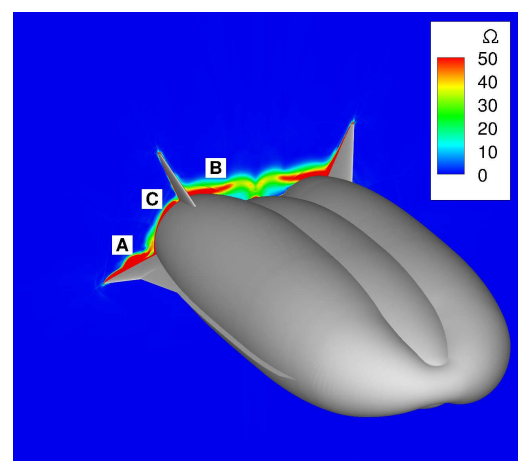

(b) $x=0.85 \mathrm{~L}, \alpha=10$ deg.

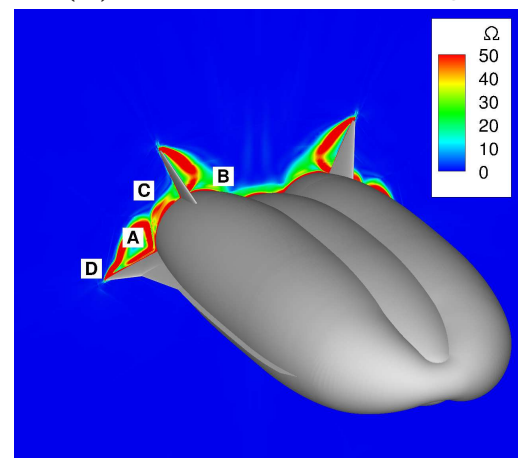

(e) $x=0.85 \mathrm{~L}, \alpha=20$ deg.

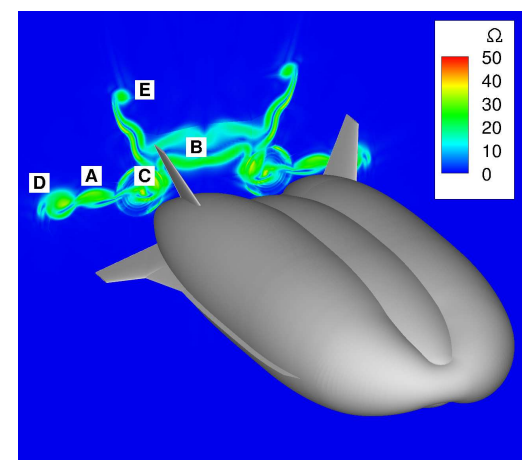

(c) $x=1.1 \mathrm{~L}, \alpha=10 \mathrm{deg}$.

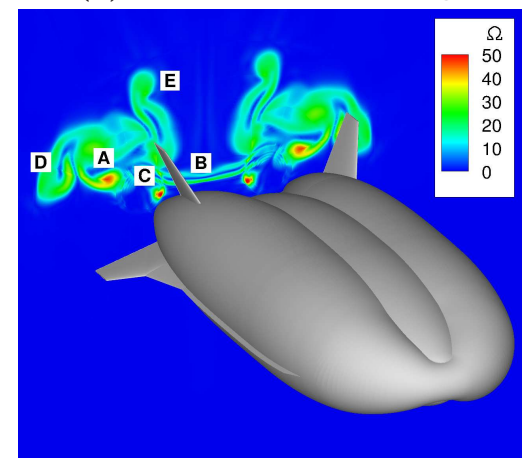

(f) $x=1.1 \mathrm{~L}, \alpha=20 \mathrm{deg}$.

Figure 7: Flow around the approximated Airlander 50 at $\alpha=10^{\circ}$ and $20^{\circ}, M_{\infty}=0.12$ and $R e=3 \cdot 10^{6}$. Contours of vorticity magnitude.

Moving further downstream at $x=0.85 \mathrm{~L}$ (Figures $\mathbf{7}$ (b) and (e)) the strake vortex (A) becomes bigger due to the presence of the LERX (that has more area), and eventually merges with the vortex generated by the lower fin (D). Second and third vortices are also generated by the hull. The second one $(\mathbf{C})$ is generated between the upper and lower fins and can be clearly identified in Figure 7 (e). This vortex is shed downstream and is seen in Figure $\mathbf{7}$ (f) with high vorticity magnitude and located outwards the fin (C). The third one is generated on the lower surface of the vehicle.

The tip of the upper fins also generate vortices (E), which can be easily distinguished in Figures 7 (c) and (f). Since the vortices of the upper and lower fins are co-rotating, they interact and eventually merge further downstream. 


\section{Transition effects}

The location of the transition onset on the Airlander 50 is estimated, employing empirical criteria on the surface pressure from fully turbulent CFD cases. For this, the Miche ${ }^{25}$ and Cebeci and Smith criteria ${ }^{26}$ are employed on streamlines extracted in the boundary layer of each of the computations. It should be noted that these criteria are not coupled in the CFD method, but are only used here to estimate the transition location. These criteria are based on experimental data on flat plates and aerofoils and provide an estimate of the location of the transition onset when the local momentum thickness Reynolds number $\left(R e_{\theta, t r}\right)$ reaches a particular value. To obtain this turbulent quantity, the following empirical equation is employed:

$$
\operatorname{Re}_{\theta, t r} \approx \frac{U_{e}(x) \theta(x)}{\nu},
$$

where the velocity at the edge of the boundary layer is obtained as $U_{e}=\sqrt{1-C_{p}}$ and $\nu$ is the dynamic viscosity. The momentum thickness $(\theta)$ is estimated with Thwaite's method 27 as,

$$
\theta^{2}=\frac{0.45 v}{U_{e}^{6}} \int_{x_{0}}^{x} U_{e}^{5} d x
$$

In the case of Michel's criterion, 25 the transition onset location takes place when

$$
R e_{\theta, t r} \approx 2.9 R e_{x, t r}^{0.4}
$$

where $R e_{x, t r}$ is the Reynolds number, based on the distance measured from the stagnation point. In Cebeci and Smith's correlation, 26 the transition is located at the point where

$$
R e_{\theta, t r}=1.174\left(1+\frac{22400}{R e_{x, t r}}\right) R e_{x, t r}^{0.46}
$$

Figure 8 (a) shows the momentum thickness Reynolds number $\left(R e_{\theta}\right)$ along the arc of the streamline of the CFD solution and the empirical criteria, where changes in Reynolds number were considered. This analysis was performed in the streamline highlighted at the top of Figure 8 (a), that extends from the nose to the back of the vehicle's upper surface and is located between 10 and $15 \%$ of the span. Figure 8 (b) shows the pressure and momentum thickness distribution along the streamline. At $R e=3$ million, the presence of favourable pressure gradients make the boundary layer to be laminar until $80 \% \mathrm{~L}$, approximately. When the Reynolds number is reduced, the boundary layer becomes less turbulent. The opposite effect happens when the Reynolds number increases. The transition onset is moved downstream and upstream, for lower and higher Reynolds respectively. When the Reynolds number was reduced by a third, the transition onset was moved downstream by $5 \% \mathrm{~L}$, approximately. When it was increased from 3 to 10 million, the transition onset moved upstream by $42 \% \mathrm{~L}$. These results show the strong effect on the Reynolds number to the nature of the boundary layer.

As can be seen in the iso-surfaces of reversed flow of Figure 9, when the Reynolds number is higher (and therefore the boundary layer is more turbulent), the region of separated flow is smaller. Conversely, at lower Reynolds number the flow tends to separate more.

\section{Study of the role of the components}

To quantify the role of each of the components of the 'AL50' airship, cases AL50 1 to AL50 4 from Table 1 are compared in this section. For this, the flow is firstly analysed and sensitivity studies on the loads and stability are provided. 


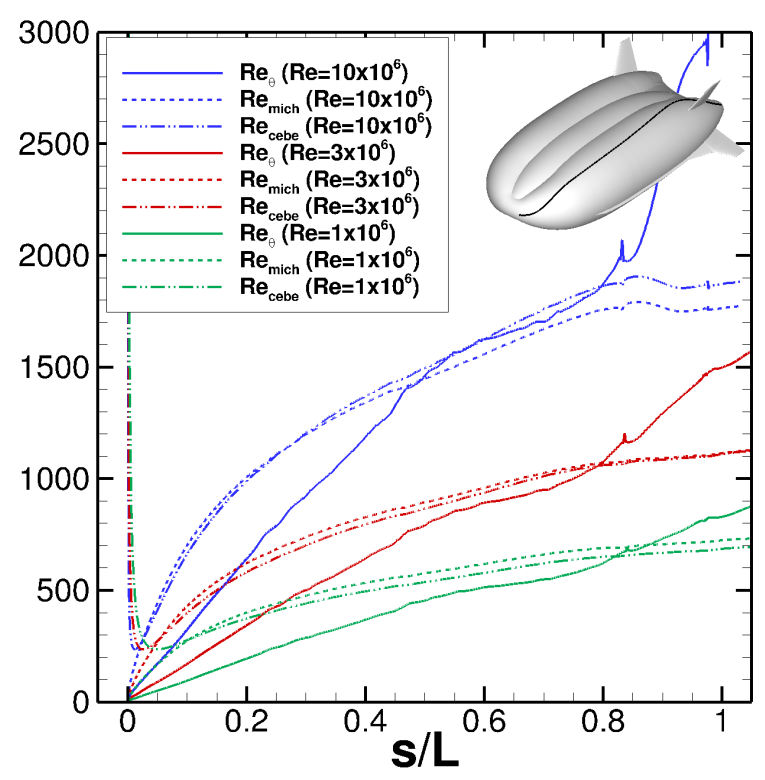

(a) Empirical transition criteria.

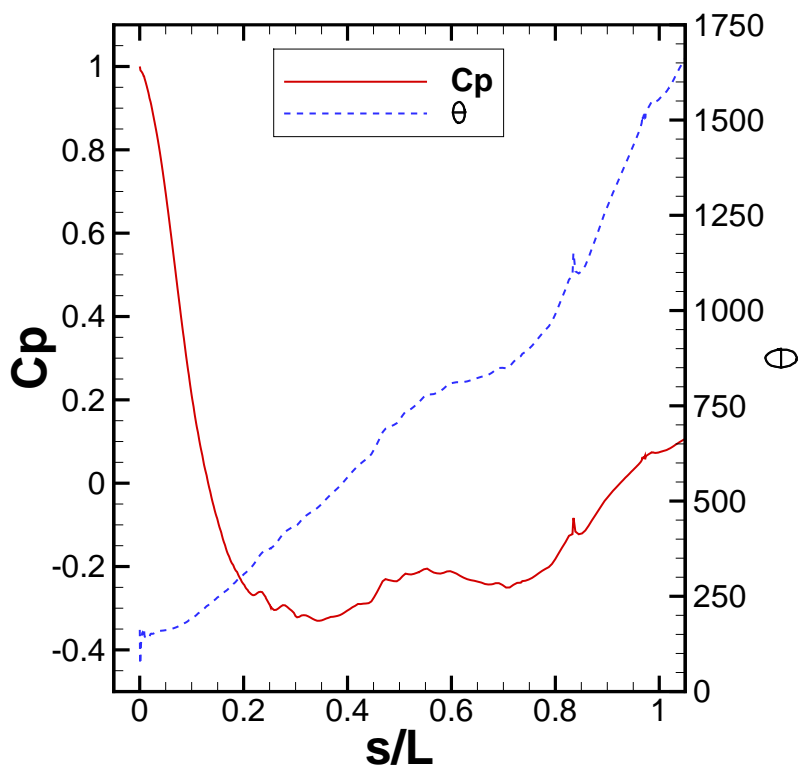

(b) Properties of the streamline.

Figure 8: Flow around the approximated Airlander 50 at $\alpha=0^{\circ}, M_{\infty}=0.12$ and $R e=1,3$, and $10 \cdot 10^{6}$. Sensitivity of the transition onset to the Reynolds number.

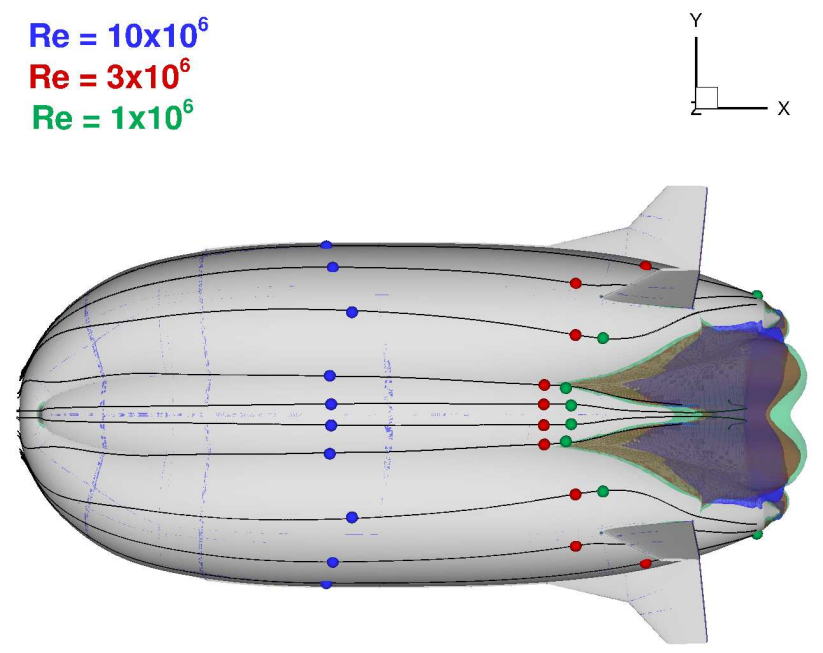

Figure 9: Flow around the approximated Airlander 50 at $\alpha=0^{\circ}, M_{\infty}=0.12$ and $R e=1,3$, and $10 \cdot 10^{6}$. Transition onset and iso-surfaces of reverse flow.

\section{Role of the airship components}

Figure 10 shows the vortex downstream of the vehicle (at $x=1.05 \mathrm{~L}$ ), with contours of vorticity magnitude $(\Omega)$, at 20 degrees of pitch. For the bare hull, only two vortices (A) are shed (Figure 10 (a)), that rotate 
clock-wise and which are due to the curvature of the hull. Two vortices are shed by the tips of the fins (one for the upper (B) and another one for the lower $(\mathbf{C})$ ), see Figure10 (b). The LERX provides an extra disturbance that merges with the one created by the lower fin $(\mathbf{C}+\mathbf{D})$, as can be seen in Figure 10 (c). The strakes generate another vortex, that blends with the one generated by the fin $(\mathbf{C}+\mathbf{D}+\mathbf{E})$, since they are co-rotating, see Figure 10 (d).

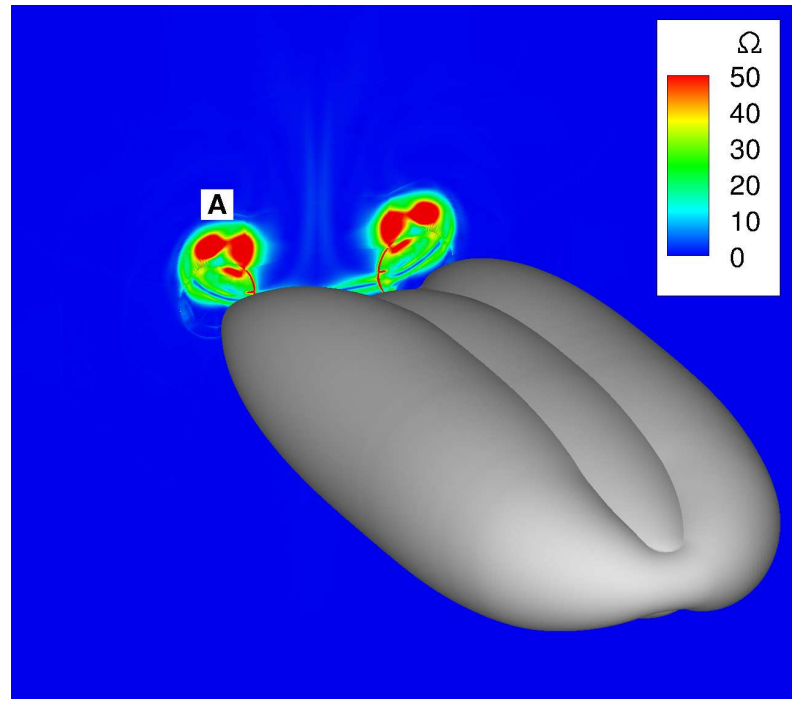

(a) Bare hull.

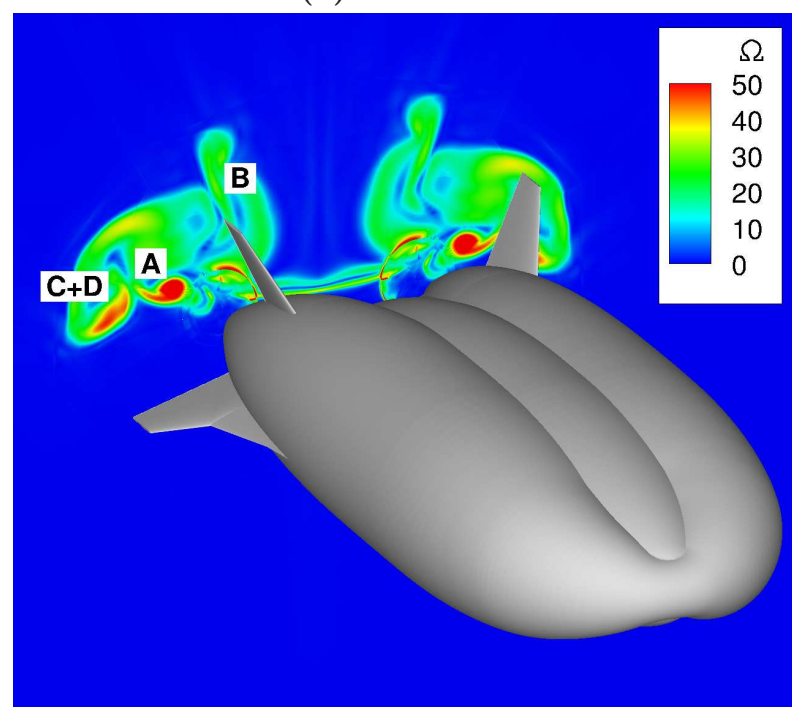

(c) Hull, fins and LERX.

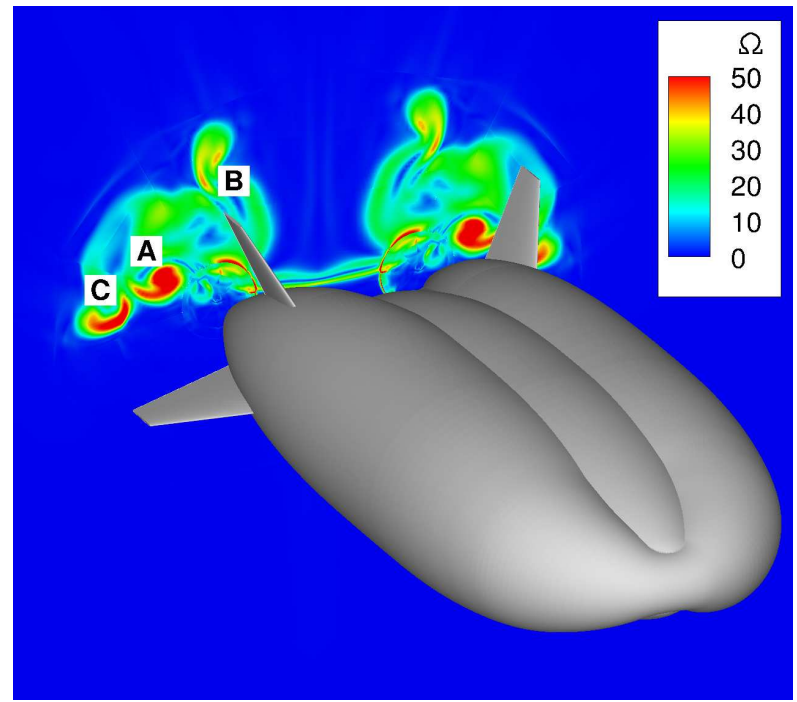

(b) Hull and fins.

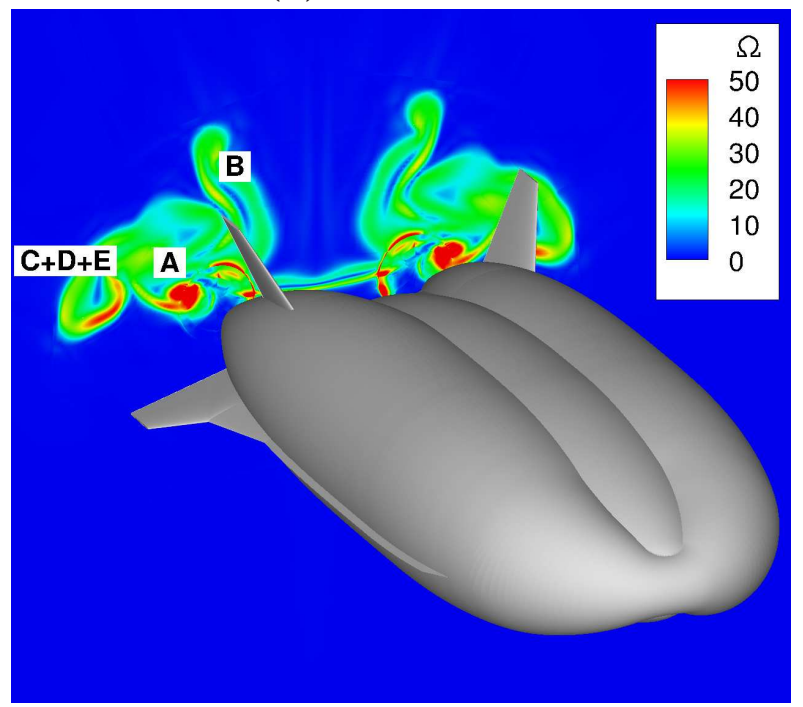

(d) Hull, fins, LERX and strakes.

Figure 10: Flow around the approximated Airlander 50 at $\alpha=20^{\circ}, M_{\infty}=0.12$ and $R e=3 \cdot 10^{6}$. Contours of vorticity magnitude for different vehicle configurations.

The vortices generated on the baseline strakes at different pitch angles (visualised with streamlines) are presented in Figure 11. At higher pitch angles the vortices are stronger and longer as observed by the streamlines. On the other hand, at negative pitch the vortex is shed towards the low side of the strake, therefore reducing the lift. 


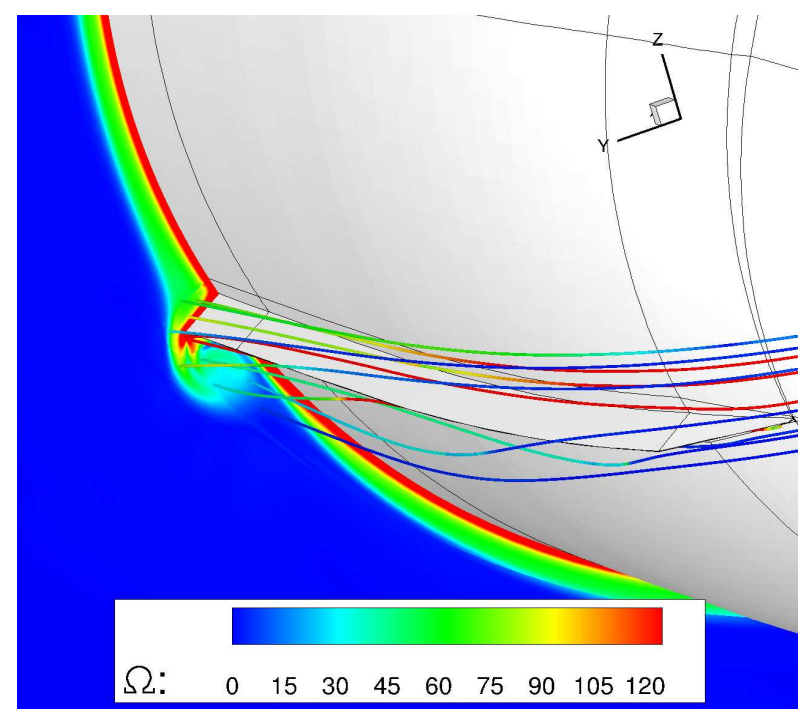

(a) $\alpha=-2$ deg.

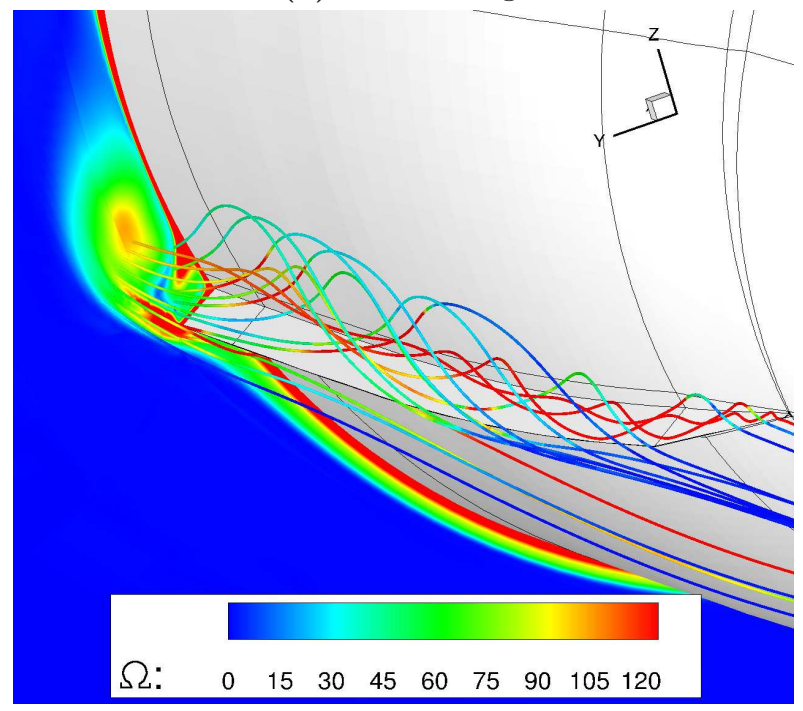

(c) $\alpha=10$ deg.

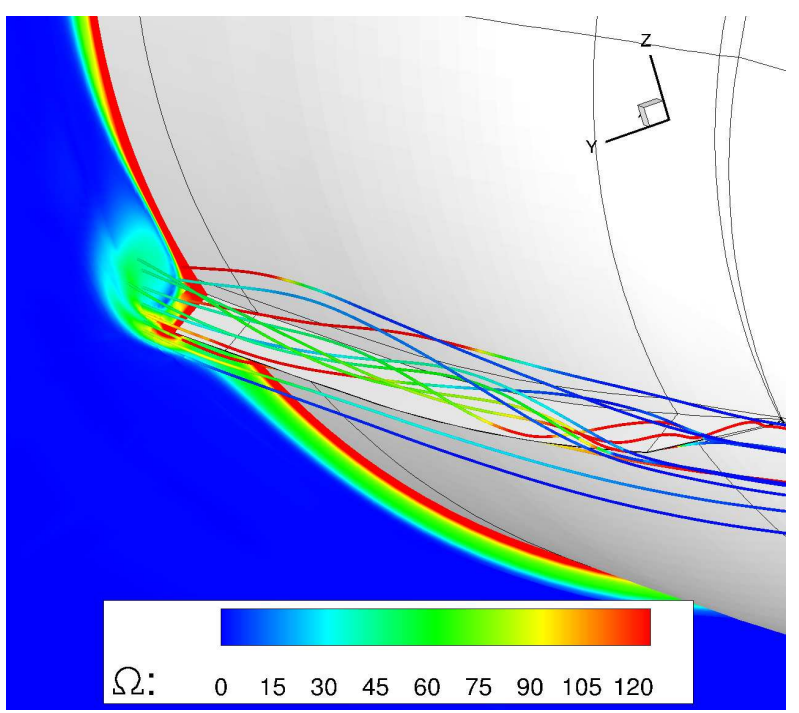

(b) $\alpha=4 \mathrm{deg}$.

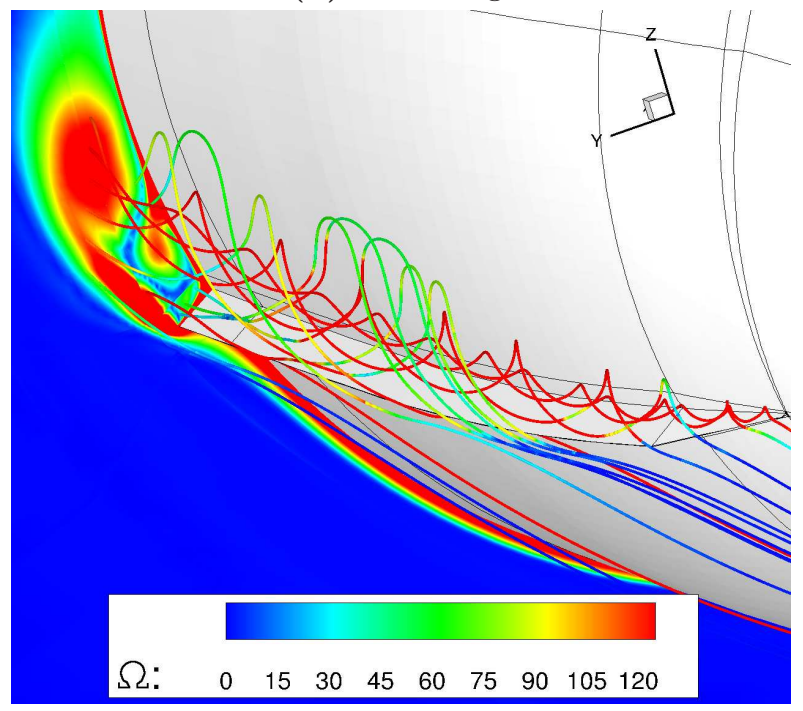

(d) $\alpha=20$ deg.

Figure 11: Flow around the approximated Airlander 50 at $\alpha=-2,4,10$ and $20^{\circ}, M_{\infty}=0.12$ and $R e=3 \cdot 10^{6}$. Vortex generated by the baseline strake visualised with flow streamlines.

\section{Role of the components on the loads and pitch stability}

Figures 12 (a) and (b) show the polars of lift and drag coefficients (based on the vehicle's volume to the 2/3 power) and Figure 12 (c) presents the lift-drag ratio. Note that all computations were run as steady-state. The highest pitch angle case ( $\alpha=20$ deg.) was computed steady and unsteady and the same aerodynamic coefficients were obtained. Figure 12 therefore shows that for the studied range of pitch angles, steady computations can be employed, since there is agreement between the RANS and URANS solutions.

For conventional and hybrid airships, the zero-lift drag coefficient $\left(C_{D V_{(H)}}\right)$ is typically estimated employing Hoerner's equation (chapter VI, eq. (36) of Hoerener's book ${ }^{28}$ ):

$$
C_{D V_{(H)}}=C_{F} \cdot\left[4 \cdot\left(\frac{l}{d}\right)^{1 / 3}+6 \cdot\left(\frac{d}{l}\right)^{1.2}+24 \kappa \cdot\left(\frac{d}{l}\right)^{2.7}\right] .
$$


In equation (7), the first term in the right hand side corresponds to the friction drag; the second term is pressure drag; the third one is the drag due to separation. For the friction coefficient $\left(C_{F}\right)$, Schoenerr's 28 formula presented in eq. (8) is employed, which is only dependent on the Reynolds number $(R e)$.

$$
C_{F}=\frac{1}{\left(3.46 \cdot \log _{10} R e-5.6\right)^{2}}
$$

It should be noted, however, that the $l / d$ ratio for airships is difficult to define, since it is not perfectly cylindrical. Here, the reference diameter $(d)$ was chosen as the equivalent one of a circle with the same area as the cross-section of the vehicle at mid-length.

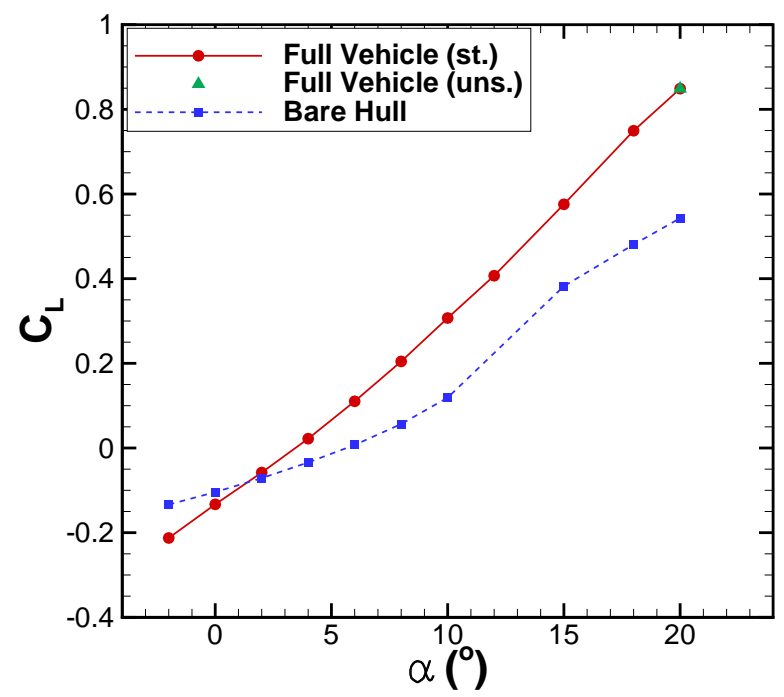

(a) Lift coefficient.

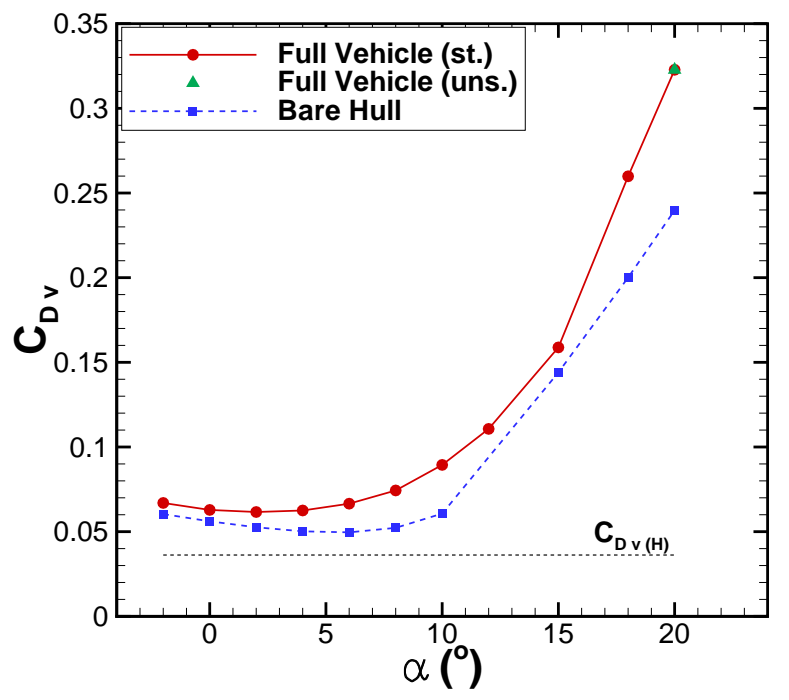

(b) Drag coefficient.

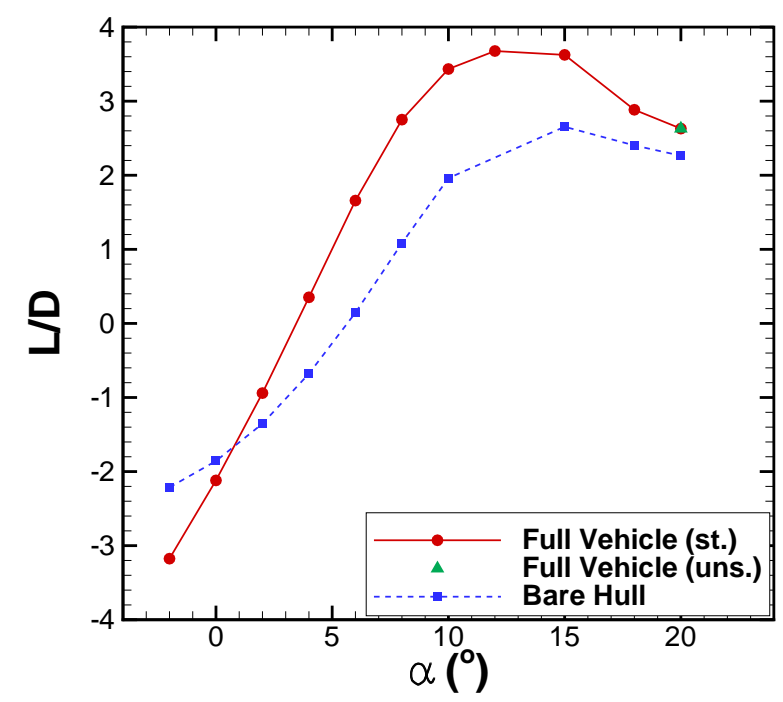

(c) $L / D$ ratio.

Figure 12: Polars of lift and drag, and $L / D$ ratio (st.: steady, uns.: unsteady).

As can be seen in Figure 12 (a), for the design employed in the present paper, zero lift is achieved at 5 deg. of pitch. For this angle, there is reasonable agreement of the drag coefficient with the theory, as shown in Figure 12 (b), although the theory gives lower drag as is strictly applied to spheroids. 
For positive angles, the components increase the lift and also the drag, which is more obvious at high pitch angles. For example, at 20 degrees of pitch, the lift and drag increased by $56.3 \%$ and $34.5 \%$, respectively. This means that at that $\alpha$, more than half of the overall lift generated by the vehicle is due to the fins, LERX and strakes. Conversely, at negative pitch angles these components contribute to reduce the lift.

The lift-drag ratio reaches a peak at $\alpha=12 \mathrm{deg}$. with a value of 2.6 for the bare hull case and 3.8 for the full vehicle. Although at high pitch angles the components increased the lift considerably, as shown in Figure 12 (a), the drag increased further (see Figure 12 (b)). The $L / D$ therefore increased by almost $160 \%$ at $\alpha=4 \mathrm{deg}$., but only by $16.5 \%$ at $\alpha=20 \mathrm{deg}$. This shows that the aerodynamic components are less effective for pitch angles higher than $12 \mathrm{deg}$.

Figure 13 shows slices at the hull's symmetry and mid-height planes for the bare hull and the full vehicle configurations, for $20 \mathrm{deg}$. of pitch. As can be observed, at the symmetry plane (Figure 13 (a)), the pressure at the hull is mainly affected by the fins at the back. For the mid-plane slice of Figure 13 (b), a significant change in the pressure is observed due to the vortex generated by the strakes that affects the boundary layer of the hull. Likewise, the LERX and fins also change the pressure profile. This can be better observed in the pressure coefficient contours of Figure 14, where there is a trace of suction on the hull due to the presence of both the LERX and strake. This shows that not only the different components contribute to a change on loads, but also the contribution of the hull itself changes, due to the interaction.

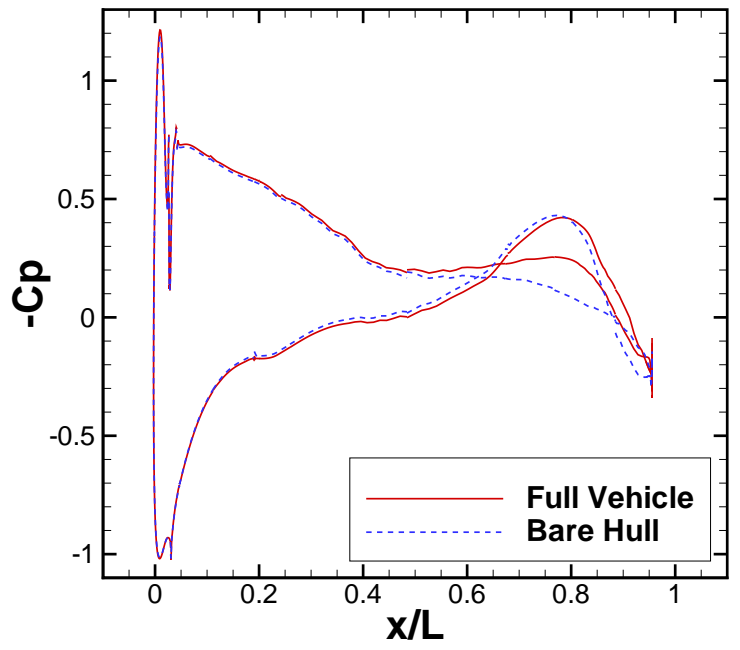

(a) Symmetry plane.

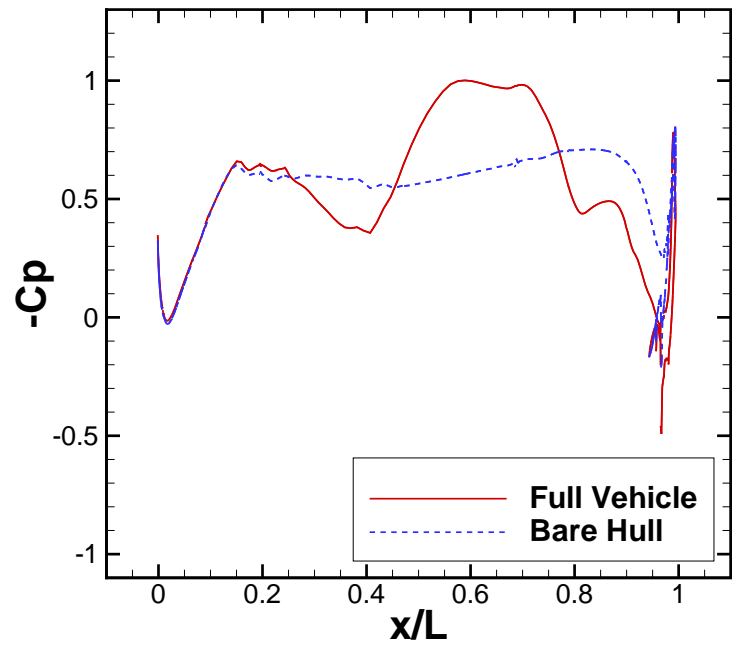

(b) Mid-height plane.

Figure 13: Effect of the components on the the pressure coefficient $\left(C_{p}\right)$ on the hull, at 20deg. of pitch.

To assess which components are the ones contributing the most to the overall lift and drag, Figure 15 shows the relative changes with respect to the bare hull case (AL50 1) for the four vehicle configurations presented in Figure 2, at 10 and 20 degrees of pitch. At 10 deg. of pitch, as presented in Figure 15 (a), the fins increased the lift by more than twice and the LERX and strakes contributed to a further small increase. The drag also increased when each component was considered, but in less percentage. As Figure 15 (b) shows, at 20 deg. of pitch, the increase in loads is not as drastic as in the previous case. The fins increased the lift by less than $50 \%$ and the strakes seem to provide more lift than the LERX. In this case, the penalties in drag were closer to the increase in drag than at in the previous case. 


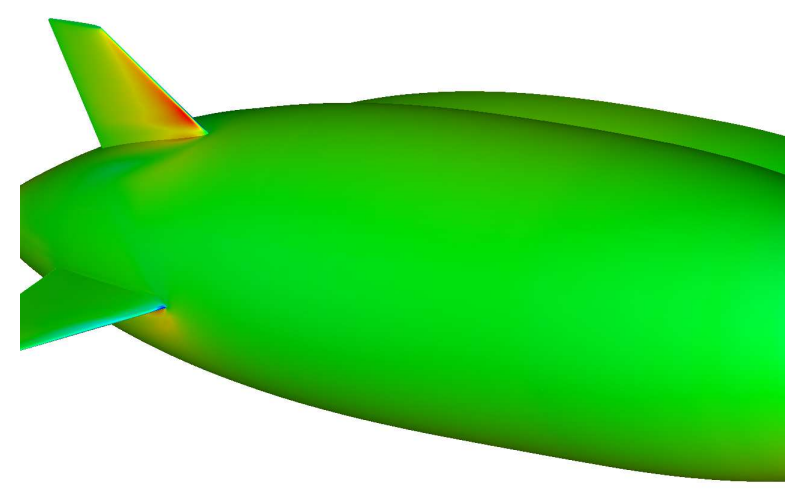

$\mathbf{C}_{\mathrm{P}}$

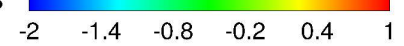

(a) Fins only.

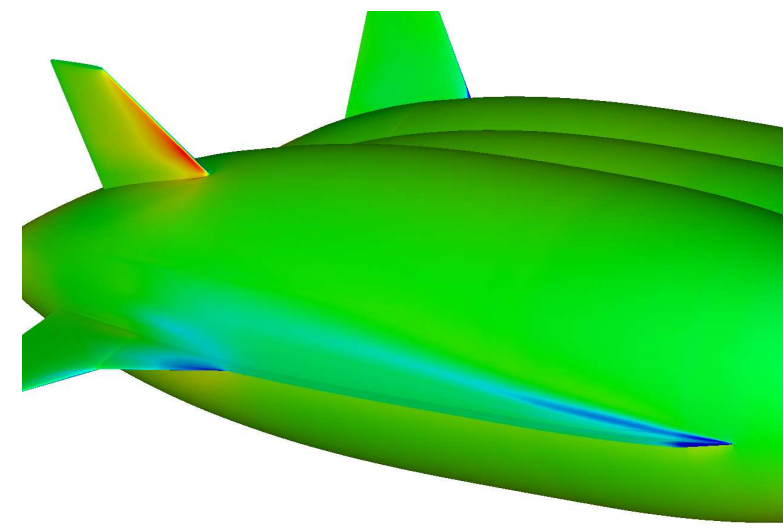

$\mathbf{C}_{\mathrm{P}}$

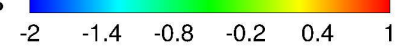

(b) Fins, LERX and strakes.

Figure 14: Contours of pressure coefficient $\left(C_{p}\right)$ on the surface of the vehicle, at 20deg. of pitch.

These results show that with the presented configuration of the approximated Airlander 50, the fins are the components that contribute the most to the lift and also drag.

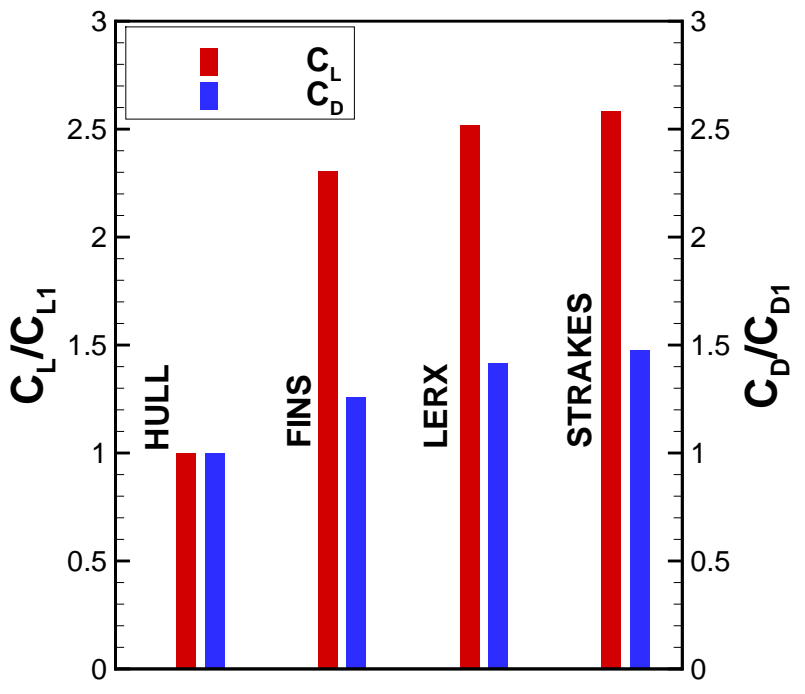

(a) $\alpha=10$ deg.

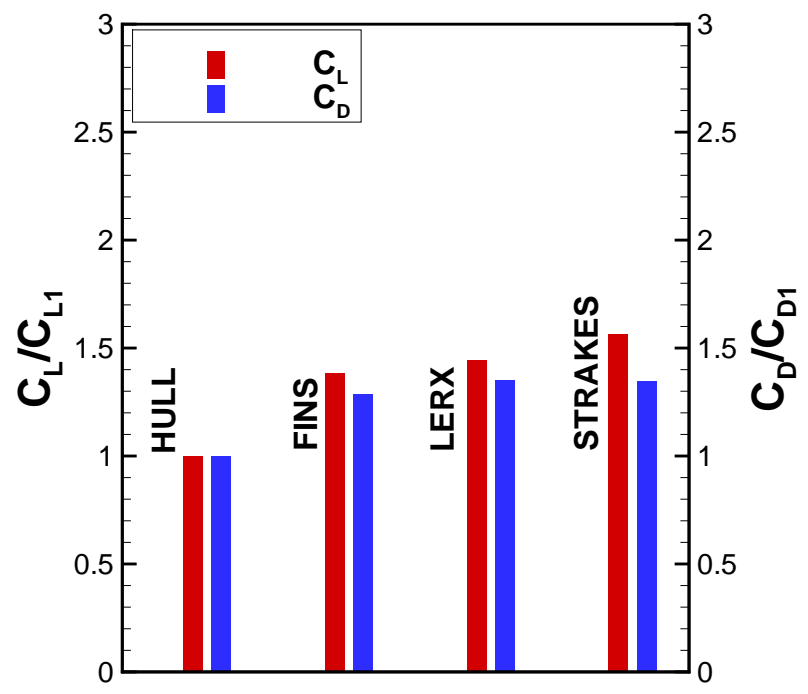

(b) $\alpha=20$ deg.

Figure 15: Contribution of each component to the lift and drag, with respect to the bare hull configuration.

Regarding the stability of the vehicle, the pitching moment $\left(C_{M_{y}}\right)$ and the change of it with the pitch angle $\left(C_{M_{\alpha}}\right)$, calculated at the center of volume and based on the vehicle's volume, are presented in Figure 16. Note that positive $C_{M_{\alpha}}$ means nose up and negative is nose down. As Figure16 (b) shows, both bare hull and full configurations seem to be unstable, as the tendency of the vehicle is to pitch up for positive changes in pitch. This should be attributed to the fact that no buoyancy force was included in the computations. Similar behaviour was observed in other works in the literature that did not account for buoyancy effects. 


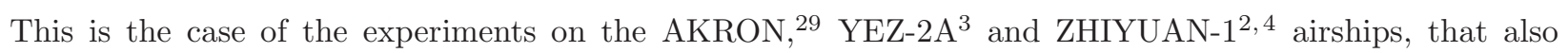
presented positive $C_{M_{\alpha}}$.

Nevertheless, the presence of the aerodynamic components have a stabilising effect, since there is a reduction in the pitch derivative. This was also seen by Wang et al ${ }^{[2}$ and Freeman ${ }^{[29}$ In addition, since the 'AL50' is not axisymmetric, at zero pitch angle the pitching moment is not zero, which differs from other more traditional single-lobe airship configurations.24 40

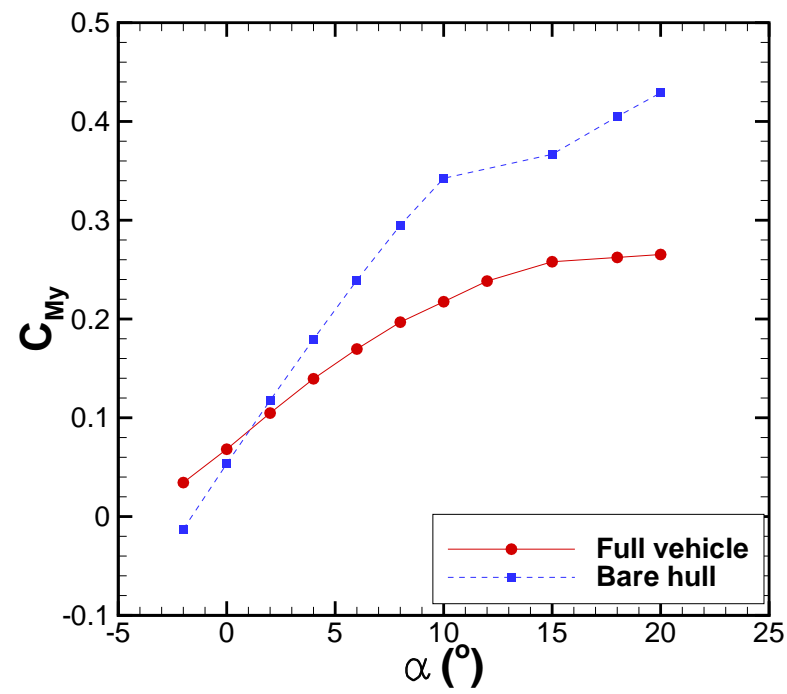

(a) Pitching moment.

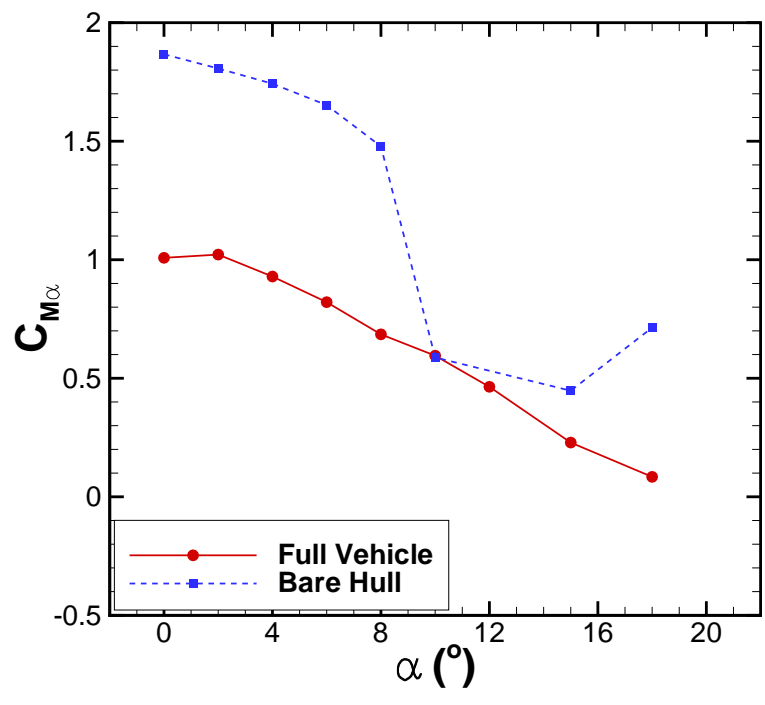

(b) Derivative of pitching moment.

Figure 16: Pitching moment $\left(C_{M_{y}}\right)$ and derivative $\left(C_{M_{\alpha}}\right)$ at the center of volume.

\section{Conclusions}

The employed CFD method (HMB2) was validated for the 6:1 prolate spheroid, and showed good agreement with the experiments.

The flow around an early design of the Airlander 50 airship was analysed, and the body vortices were identified. Due to the presence of favourable pressure gradients, the onset of transition was predicted close to the back of the airship, at $80 \% \mathrm{~L}$ approximately. Sensitivity studies on the Reynolds number showed a further downstream transition onset for lower Reynolds numbers and an increase of Reynolds led to an earlier transition onset.

The study continued by exploring the role of each component of the airship on the aerodynamic coefficients and the stability derivatives. The results showed that with the standard vehicle configuration, the fins contributed the most to an increase in lift and also drag.

In the future, the effect of the propulsion elements on the flow is to be investigated.

\section{Acknowledgements}

The computations were performed on the N8 HPC facility "Polaris" at Leeds University and the Chadwick cluster of the University of Liverpool. Access to both systems is gratefully acknowledged, as well as the financial support by the LOCATE Project. 


\section{References}

${ }^{1}$ Carichner, G. and Nicolai, L., "Hybrids...the Airship Messiah?" Proceedings of the AIAA Lighter-Than-Air Systems Technology (LTA) Conference, AIAA Paper 2013-1317, Daytona Beach, Florida, USA, March 2013.

${ }^{2}$ Wang, X., Fu, G., D, P., and Shan, X., "Experimental Investigations on Aerodynamic Characteristics of the ZHIYUAN-1 Airship," Journal of Aircraft, Vol. 47, No. 4, July-August 2010, pp. 1463-1468.

${ }^{3}$ Gomes, S., "An Investigation of the Flight Dynamics of Airships with Application to the YEZ-2A," PhD Thesis, Cranfield Institute of Technology, Cranfield, England, UK, October 1990.

${ }^{4}$ Suman, S., Lakshmipathy, S., and Pant, R., "Evaluation of Assumed-Transition-Point Criterion in Context of ReynoldsAveraged Simulations Around Lighter-Than-Air Vehicles," Journal of Aircraft, Vol. 50, No. 2, March-April 2013, pp. 450-456.

${ }^{5}$ Dumas, A., Madonia, M., Ilieva, G., Trancossi, M., Pascoa, J., and Coppola, A., "CFD Analysis and Optimization of a Variable Shape Airship," Proceedings of the ASME 2012 International Mechanical Engineering Congress 8 Exposition, American Soc. of Mechanical Engineers Paper IMECE2012-87375, Fairfield, NJ, November 2012.

${ }^{6}$ Wetzel, T., "Unsteady Flow Over a 6:1 Prolate Spheroid," PhD Thesis, Virginia Polytechnic Institute and State University, 1996.

${ }^{7}$ Clarke, D., Brandner, P., and Walker, G., "Experimental and Computational Investigation of Flow Around a 3:1 Prolate Spheroid," WSEAS Transactions of Fluid Mechanics, Vol. 3, No. 3, July 2008, pp. 207-217.

${ }^{8}$ Sorensen, N., "CFD Modelling of Laminar-Turbulent Transition for Airfoils and Rotors Using the $\gamma-\operatorname{Re}_{\theta}$ Model," Wind Energy, Vol. 12, April 2009, pp. 715-733.

${ }^{9}$ Vatsa, V., Thomas, J., and Wedan, B., "Navier-Stokes Computations of a Prolate Spheroid at Angle of Attack," Journal of Aircraft, Vol. 26, No. 11, 1989, pp. 986-993.

${ }^{10}$ Constantinescu, G., Pasinato, H., Wang, Y., Forsythe, J., and Squires, K., "Numerical Investigation of Flow Past a Prolate Spheroid," Transactions of the ASME, Vol. 124, No. 6, December 2002, pp. 904-910.

${ }^{11}$ Omari, K., Schall, E., Koobus, B., and Dervieux, A., "Turbulence Modeling Challenges in Airship CFD Studies," Monografías del Seminario Matemático García Galdeano, Vol. 31, 2004, pp. 545-554.

${ }^{12}$ Omari, K., Schall, E., Le Guer, Y., and Amara, M., "Numerical Study of Turbulent Flow Around a Generic Airship at High Angle of Attack," 4th International Conference on Computational Heat and Mass Transfer, Paper 372, École normale supérieure de Cachan, Paris, May 2005.

${ }^{13}$ Barakos, G., Steijl, R., and Badcock, K., "Development of CFD Capability for Full Helicopter Engineering Analysis," 31st European Rotorcraft Forum [CD-ROM], Paper 91, Council of European Aerospace Soc., Florence, Italy, September 2010.

${ }^{14}$ Osher, S. and Chakravarthy, S., "Upwind Schemes and Boundary Conditions with Applications to Euler Equations in General Geometries," Journal of Computational Physics, Vol. 50, No. 3, June 1983, pp. 447-481.

${ }^{15}$ van Leer, B., "Towards the Ultimate Conservative Difference Scheme, V. A Second Order Sequel to Godunov's Method," J. Comp. Phys., Vol. 32, No. 1, 1979, pp. 101-136.

${ }^{16}$ Rieper, F., "A low-Mach number fix for Roe's approximate Riemann solver," Journal of Computational Physics, Vol. 230, No. 13, March 2011, pp. 5263-5287.

${ }^{17}$ Carrión, M., Woodgate, M., Steijl, R., and Barakos, G., "Implementation of All-Mach Roe-type Schemes in Fully Implicit CFD Solvers - Demonstration for Wind Turbine Flows," International Journal for Numerical Methods in Fluids, Vol. 73, No. 8, 2013, pp. 693-728.

${ }^{18}$ Axelsson, O., Iterative Solution Methods, Cambridge University Press: Cambridge, MA, pp. 504-557, 1994.

${ }^{19}$ Menter, F. and Egorov, Y., "The Scale-Adaptive Simulation Method for Unsteady Turbulent Flow Predictions. Part 1: Theory and Model Description," Flow Turbulence Combust, Vol. 85, No. 1, 2010, pp. 113-138.

${ }^{20}$ Zografakis, G. and Barakos, G., "Transition Modelling for Rotorcraft CFD," 34th European Rotorcraft Forum, Liverpool, UK, Paper 5b3, Royal Aeronautical Soc. London, September 2008.

${ }^{21}$ Steijl, R. and Barakos, G., "Sliding Mesh Algorithm for CFD Analysis of Helicopter Rotor-Fuselage Aerodynamics," Int. J. Numer. Meth. Fluids, Vol. 58, No. 5, October 2008, pp. 527-549.

${ }^{22}$ Chesnakas, C. and Simpson, R., "Detailed Investigation of the Three-Dimensional Separation About 6:1 Prolate Spheroid," AIAA Journal, Vol. 35, No. 6, June 1997, pp. 990-999.

${ }^{23}$ Menter, F., "Two-Equation Eddy-Viscosity Turbulence Models for Engineering Applications," AIAA Journal, Vol. 32, No. 8, 1994, pp. 1598-1605.

${ }^{24}$ Jeong, J. and Hussain, F., "On the Identification of a Vortex," Journal of Fluid Mechanics, Vol. 285, February 1995, pp. 69-94. 
${ }^{25}$ Michel, R., Etude de la transition sur les profils d'aile, etablissement d'un critere de determination du point de transition et calcul de la trainee de profil incompressible, ONERA TR 1/1578A, Paris, 1951.

${ }^{26}$ Cebeci, T. and Smith, A. M. O., Analysis of Turbulent Boundary Layers, No. 15, Applied Mathematics and Mechanics. Academic Press, New York, NY, 1974.

${ }^{27}$ Thwaite, B., "Approximate Calculation of the Laminar Boundary Layer," Aeronautical Quarterly, Vol. 1, 1949, pp. 245280.

${ }^{28}$ Hoerner, S., Fluid-dynamic drag; practical information on aerodynamic drag and hydrodynamic resistance, Midland Park, N. J., Chap 6, 1958.

${ }^{29}$ Freeman, H., "Force Measurements on a 1/40-scale Model of the U.S. Airship 'AKRON'," PhD Thesis, Cranfield Institute of Technology, Cranfield, England, UK, October 1990.

${ }^{30}$ Funk, P., Lutz, T., and Wagner, S., "Experimental Investigations on Hull-Fin Interferences of the Lotte Airship," Aerospace Science and Technology, Vol. 7, No. 8, 2003, pp. 603-610. 